\title{
TITLE:
}

\section{A modified melting crystal model and the Ablowitz-Ladik hierarchy}

$\operatorname{AUTHOR}(\mathrm{S})$ :

Takasaki, Kanehisa

\section{CITATION:}

Takasaki, Kanehisa. A modified melting crystal model and the Ablowitz-Ladik hierarchy. Journal of Physics A: Mathematical and Theoretical 2013, 46(24): 245202

ISSUE DATE:

2013-05-24

URL:

http://hdl.handle.net/2433/175510

\section{RIGHT:}

(C) 2013 IOP Publishing.; この論文は出版社版でありません。引用の際 には出版社版をご確認ご利用ください。; This is not the published version. Please cite only the published version. 


\title{
Modified melting crystal model and Ablowitz-Ladik hierarchy
}

\author{
Kanehisa Takasaki* \\ Graduate School of Human and Environmental Studies \\ Kyoto University \\ Sakyo, Kyoto 606-8501, Japan
}

\begin{abstract}
This paper addresses the issue of integrable structure in a modified melting crystal model of topological string theory on the resolved conifold. The partition function can be expressed as the vacuum expectation value of an operator on the Fock space of $2 \mathrm{D}$ complex free fermion fields. The quantum torus algebra of fermion bilinears behind this expression is shown to have an extended set of "shift symmetries". They are used to prove that the partition function (deformed by external potentials) is essentially a tau function of the $2 \mathrm{D}$ Toda hierarchy. This special solution of the $2 \mathrm{D}$ Toda hierarchy can be characterized by a factorization problem of $\mathbb{Z} \times \mathbb{Z}$ matrices as well. The associated Lax operators turn out to be quotients of first order difference operators. This implies that the solution of the 2D Toda hierarchy in question is actually a solution of the Ablowitz-Ladik (equivalently, relativistic Toda) hierarchy. As a byproduct, the shift symmetries are shown to be related to matrix-valued quantum dilogarithmic functions.
\end{abstract}

Pacs Numbers: 02.30.Ik, 11.25.Hf

2010 Mathematics Subject Classification: 17B65, 35Q55, 81T30, 82B20 Key words: melting crystal, topological string, resolved conifold, free fermion, quantum torus, shift symmetry, quantum dilogarithm, Toda hierarchy, Ablowitz-Ladik hierarchy

*takasaki@math.h.kyoto-u.ac.jp 


\section{Introduction}

This is a sequel of our previous work $[1,2]$ on the integrable structure of the "melting crystal model" of topological string theory [3] and 5D $\mathcal{N}=1$ supersymmetric $U(1)$ gauge theory [4]. We here address the same issue for a modified melting crystal model that is related to topological string theory on the resolved conifold. In particular, we present an affirmative answer to a conjecture in our preliminary study on this issue [5]. In the perspectives of topological string theory, the partition functions of these two models are the simplest non-trivial cases of the topological vertex construction [6] of string amplitudes on non-compact Calabi-Yau 3-folds. The Calabi-Yau 3-folds relevant to our models are the first two members of the so called "local $\mathbb{C P}^{1}$ geometries" $X_{l}=\mathcal{O}(-l) \oplus \mathcal{O}(l-2) \rightarrow \mathbb{C P}^{1}, l=0,1, \ldots$ The resolved conifold amounts to the case of $l=1$. Local Gromov-Witten invariants of these manifolds are studied by the localization technique [7]. Generating functions of these topological invariants coincide with the topological string amplitudes obtained by the method of topological vertex. Moreover, the genus-zero part of those invariants is determine by the random matrix technique [8].

The partition function $Z$ of the previous model, see (2.1), is a sum of weights labelled by integer partitions of arbitrary lengths. This statistical sum is derived from the partition function of random plane partitions (3D Young diagrams) by the method of diagonal slicing [9]. The aforementioned integrable structure $[1,2]$ emerges when $Z$ is deformed by external potentials. The partition function of the deformed model is a function $Z(s, \boldsymbol{t})$ of a discrete variable $s$ and a set of continuous variables $\boldsymbol{t}=\left(t_{1}, t_{2}, \ldots\right) . s$ is a parameter of the external potentials and $t_{k}$ 's are coupling constants. The deformed partition function $Z(s, \boldsymbol{t})$ turns out to be related to a tau function $\tau(s, \boldsymbol{t})$ of the $1 \mathrm{D}$ reduction of the 2D Toda hierarchy $[10,11] . s$ and $\boldsymbol{t}$ play the role of a lattice coordinate and time variables in the 1D Toda hierarchy. The relation to the Toda hierarchy is explained with the aid of $2 \mathrm{D}$ complex free fermion fields that are extensively used for integrable hierarchies as well $[12,13] . Z(s, \boldsymbol{t})$ can be thereby expressed as the vacuum expectation value of an operator in the fermion Fock space. We found that this fermionic expression of $Z(s, \boldsymbol{t})$ can be converted to the tau function $\tau(s, \boldsymbol{t})$ by a change of variables and a simple multiplicative factor. A technical clue therein is the notion of "shift symmetries" [1,2] generated by the adjoint action of special "vertex operators" [9] on the quantum torus algebra of fermion bilinears.

Our approach to the partition function $Z^{\prime}$ of the modified model, see (2.7), is mostly parallel, but has a new feature as well. Namely, $Z^{\prime}$ is deformed by two sets, rather than a single set, of external potentials. The deformed partition function is a function $Z^{\prime}(s, \boldsymbol{t}, \overline{\boldsymbol{t}})$ of a discrete parameter $s$ and coupling 
constants $\boldsymbol{t}=\left(t_{1}, t_{2}, \ldots\right)$ and $\overline{\boldsymbol{t}}=\left(\bar{t}_{1}, \bar{t}_{2}, \ldots\right)^{1}$ of the external potentials. We introduce an extended set of shift symmetries (related to vertex operators of another type [14]), and show that the deformed partition function $Z^{\prime}(s, \boldsymbol{t}, \overline{\boldsymbol{t}})$ is related to a tau function $\tau^{\prime}(s, \boldsymbol{t}, \overline{\boldsymbol{t}})$ of the 2D Toda hierarchy in the same way as $Z(s, \boldsymbol{t})$ is related to $\tau(s, \boldsymbol{t})$. It is natural to expect that this tau function, too, is a solution of a reduced system of the 2D Toda hierarchy. This is indeed the case. As we conjectured in the preliminary study [5], we here prove that $\tau^{\prime}(s, \boldsymbol{t}, \overline{\boldsymbol{t}})$ is a solution of the Ablowitz-Ladik hierarchy [15] or, speaking more fairly, the relativistic Toda hierarchy [16]. We expect that this explains an origin of the integrable structure that Brini [17] observed in the generating function of local Gromov-Witten invariants of the resolved conifold by a genus-by-genus analysis.

The Ablowitz-Ladik hierarchy is a spatial discretization of the nonlinear Schrödinger hierarchy. A traditional Lax formalism is based on a $2 \times 2$ matrix-valued zero-curvature equations [15]. A bilinear formalism consists of Hirota equations for three tau functions $\tau, \sigma$ and $\bar{\sigma}$ [18]. In the context of random matrices, a semi-infinite lattice version emerges in the unitary matrix model and the associated biorthogonal Laurent polynomials [19, 20]. Moreover, as pointed out by Kharchev et al. [21] and Suris [22], the AblowitzLadik hierarchy is equivalent to the relativistic Toda hierarchy. It is rather the relativistic Toda hierarchy that is directly connected with the 2D Toda hierarchy ${ }^{2}$. Brini et al. [24] characterized this integrable hierarchy (which they call the Ablowitz-Ladik hierarchy) as a kind of "rational reduction" of the 2D Toda hierarchy. The notion of rational reduction was first introduced in the case of the KP hierarchy [25]. In the Toda version, the Lax operators $L$ and $\bar{L}$ of the reduced system are expressed as "quotients" of finite order difference operators in the variable $s$.

We show that the Lax operators of the solution in question do have such a factorized form. To this end, we consider a factorization problem of $\mathbb{Z} \times \mathbb{Z}$ matrices $[26,27,28]$ that can capture all solutions of the 2D Toda hierarchy. In general, solving this factorization problem directly is extremely difficult. In the present case, however, we can find an explicit form of the solution at the "initial time" $\boldsymbol{t}=\overline{\boldsymbol{t}}=\mathbf{0}$. This is enough to determine the "initial values" of the Lax operators. As expected, the initial values turn out to have the factorized form of Brini et al. Since the factorized form is preserved by the flows of the 2D Toda hierarchy (this is what the notion of reduction means),

\footnotetext{
${ }^{1}$ The bars "-" do not mean complex conjugation.

${ }^{2}$ Bruschi and Ragnisco [23] proposed a Lax formalism of the relativistic Toda lattice that is based on a scalar-valued auxiliary linear problem. This auxiliary linear problem is a variant of the well known auxiliary linear problem of the usual Toda lattice, and fits into the Lax formalism of the 2D Toda hierarchy.
} 
we can conclude that the solution in question belongs to the Ablowitz-Ladik or relativistic Toda hierarchy.

Let us emphasize that shift symmetries are interesting in themselves. This is another subject of this paper. We shall encounter a few new aspects in the course of the consideration on the integrable structure. Firstly, we derive the shift symmetries more carefully than in our previous work [1, 2]. This explains why a careless use of these symmetries leads to a contradictory result [5]. Secondly, we translate the shift symmetries to the language of $\mathbb{Z} \times \mathbb{Z}$ matrices. Remarkably, this reveals a close relationship with quantum dilogarithmic functions $[29,30]$. In the matrix representation, the vertex operators $[9,14]$ in the fermionic expression of $Z(s, \boldsymbol{t})$ and $Z^{\prime}(s, \boldsymbol{t}, \overline{\boldsymbol{t}})$ turn into matrix-valued quantum dilogarithmic functions. The shift symmetries thereby become a straightforward consequence of an infinite product formula of the quantum dilogarithmic functions. We believe that this issue deserve to be studied in a more genera context.

This paper is organized as follows. Sections 2, 3, and 4 presents the perspective from fermions and tau functions. In Section 2, the partition functions of the two melting crystal models are formulated. The setup of the 2D complex free fermion system is also explained here. In Section 3, the quantum torus algebra of fermion bilinears is introduced, and the extended set of shift symmetries are derived. These algebraic relations are used in Section 4 to derive the tau functions from the deformed partition functions of the melting crystal models. Sections 5 and 6 are mostly concerned with infinite matrices and Lax equations. In Section 5, the shift symmetries are reformulated in the language of infinite matrices. This is a place where matrix-valued quantum dilogarithmic functions show up. Although the matrix versions of shift symmetries are not necessary for the subsequent consideration, the proof of these algebraic relations overlaps with technical details of the contents of Section 6 . Section 6 is devoted to the proof of our conjecture that the modified melting crystal model gives a solution of the Ablowitz-Ladik hierarchy. The relevant matrix factorization problem is formulated and solved here. The associated Lax operators are thereby shown to take a factorized form as expected.

\section{Melting crystal models}

\subsection{Previous model}

The partition function of the undeformed model is the sum

$$
Z=\sum_{\lambda \in \mathcal{P}} s_{\lambda}\left(q^{-\rho}\right)^{2} Q^{|\lambda|}, \quad|\lambda|=\sum_{i \geq 1} \lambda_{i}
$$


over the set $\mathcal{P}$ of all partitions $\lambda=\left(\lambda_{1}, \lambda_{2}, \ldots\right) . q$ and $Q$ are complex numbers with $|q|,|Q|<1$ (or may be thought of as formal variables). $s_{\lambda}\left(q^{-\rho}\right)$ is the special value of the Schur function $s_{\lambda}(\boldsymbol{x})$ of infinite variables $\boldsymbol{x}=\left(x_{1}, x_{2}, \ldots\right)$ at

$$
q^{-\rho}=\left(q^{1 / 2}, q^{3 / 2}, \ldots, q^{n-1 / 2}, \ldots\right) .
$$

This special value has the well known hook formula [31]:

$$
s_{\lambda}\left(q^{-\rho}\right)=\frac{q^{-\kappa(\lambda) / 4}}{\prod_{(i, j) \in \lambda}\left(q^{-h(i, j) / 2}-q^{h(i, j) / 2}\right)},
$$

where $\kappa(\lambda)$ are the second Casimir value (equivalently, twice the total content)

$$
\kappa(\lambda)=\sum_{i=1}^{\infty} \lambda_{i}\left(\lambda_{i}-2 i+1\right)=2 \sum_{(i, j) \in \lambda}(j-i)
$$

and $h(i, j)$ is the hook length of the cell $(i, j)$ in the Young diagram of shape $\lambda$. By the Cauchy identity,

$$
\sum_{\lambda \in \mathcal{P}} s_{\lambda}\left(x_{1}, x_{2}, \ldots\right) s_{\lambda}\left(y_{1}, y_{2}, \ldots\right)=\prod_{i, j \geq 1}\left(1-x_{i} y_{j}\right)^{-1}
$$

one can rewrite the sum $Z$ to an infinite product:

$$
Z=\prod_{i, j=1}^{\infty}\left(1-Q q^{i+j-1}\right)^{-1}=\prod_{n=1}^{\infty}\left(1-Q q^{n}\right)^{-n}
$$

We deform (2.1) with a discrete variable $s \in \mathbb{Z}$ and an infinite number of continuous variables $\boldsymbol{t}=\left(t_{1}, t_{2}, \ldots\right)$ as

$$
Z(s, \boldsymbol{t})=\sum_{\lambda \in \mathcal{P}} s_{\lambda}\left(q^{-\rho}\right)^{2} Q^{|\lambda|+s(s+1) / 2} e^{\Phi(\lambda, s, \boldsymbol{t})},
$$

where $\Phi(\lambda, s, \boldsymbol{t})$ is a linear combination

$$
\Phi(\lambda, s, \boldsymbol{t})=\sum_{k=1}^{\infty} t_{k} \Phi_{k}(\lambda, s)
$$

of the external potentials

$$
\begin{aligned}
\Phi_{k}(\lambda, s) & =\sum_{i=1}^{\infty} q^{k\left(\lambda_{i}+s-i+1\right)}-\sum_{i=1}^{\infty} q^{k(-i+1)} \quad \text { (formal expression) } \\
& =\sum_{i=1}^{\infty}\left(q^{k\left(\lambda_{i}+s-i+1\right)}-q^{k(s-i+1)}\right)+\frac{1-q^{k s}}{1-q^{k}} q^{k} .
\end{aligned}
$$

The formal expression in the first line is related to the normal ordering prescription in the fermionic expression (2.14) of these potentials. 


\subsection{Modified model}

In the modified model, one of the factors of the weight is modified as $s_{\lambda}\left(q^{-\rho}\right) \rightarrow$ $s_{{ }^{t} \lambda}\left(q^{-\rho}\right)$, where ${ }^{\mathrm{t}} \lambda$ is the conjugate (or transposed) partition of $\lambda$. Thus the undeformed partition function reads

$$
Z^{\prime}=\sum_{\lambda \in \mathcal{P}} s_{\lambda}\left(q^{-\rho}\right) s_{{ }_{t}}\left(q^{-\rho}\right) Q^{|\lambda|} .
$$

By the dual Cauchy identity

$$
\sum_{\lambda \in \mathcal{P}} s_{\lambda}\left(x_{1}, x_{2}, \ldots\right) s_{{ }{ }_{\lambda}}\left(y_{1}, y_{2}, \ldots\right)=\prod_{i, j=1}^{\infty}\left(1+x_{i} y_{j}\right),
$$

$Z^{\prime}$ can be cast into an infinite product:

$$
Z^{\prime}=\prod_{i, j=1}^{\infty}\left(1+Q q^{i+j-1}\right)=\prod_{n=1}^{\infty}\left(1+Q q^{n}\right)^{n} .
$$

Since $s_{t_{\lambda}}\left(q^{-\rho}\right)$ and $s_{\lambda}\left(q^{-\rho}\right)$ are related as

$$
s_{{ }_{\mathrm{t}}}\left(q^{-\rho}\right)=q^{\kappa(\lambda) / 2} s_{\lambda}\left(q^{-\rho}\right) .
$$

by the hook formula (2.2), one can rewrite the sum (2.7) as

$$
Z^{\prime}=\sum_{\lambda \in \mathcal{P}} s_{\lambda}\left(q^{-\rho}\right)^{2} q^{\kappa(\lambda) / 2} Q^{|\lambda|}
$$

Thus $Z$ and $Z^{\prime}$ are the first two members of the infinite family

$$
Z_{l}=\sum_{\lambda \in \mathcal{P}} s_{\lambda}\left(q^{-\rho}\right)^{2} q^{l \kappa(\lambda) / 2} Q^{|\lambda|}, \quad l=0,1, \ldots,
$$

of statistical models. $Z_{l}$ is the generating function of Gromov-Witten invariants in the local $\mathbb{C P}^{1}$ geometry $X_{l}=\mathcal{O}(-l) \oplus \mathcal{O}(l-2) \rightarrow \mathbb{C P}^{1}[7] . \quad Z$ and $Z^{\prime}$ can also be derived by the method of topological vertex [6] as the amplitudes of topological string theory on $X_{0}$ and $X_{1}$. Note, however, that the parameter $Q$ in the string amplitude of $X_{1}$ is flipped to $-Q$ [8]. This difference affects the final result (see Section 6) on the underlying integrable structure.

We now introduce two sets of continuous variables $\boldsymbol{t}=\left(t_{1}, t_{2}, \ldots\right)$ and $\overline{\boldsymbol{t}}=\left(\bar{t}_{1}, \bar{t}_{2}, \ldots\right)$, and deform the sum $(2.7)$ as

$$
Z^{\prime}(s, \boldsymbol{t}, \overline{\boldsymbol{t}})=\sum_{\lambda \in \mathcal{P}} s_{\lambda}\left(q^{-\rho}\right) s_{{ }_{\lambda}}\left(q^{-\rho}\right) Q^{|\lambda|+s(s+1) / 2} e^{\Phi(\lambda, s, \boldsymbol{t}, \overline{\boldsymbol{t}})},
$$


where $\Phi(\lambda, s, \boldsymbol{t}, \overline{\boldsymbol{t}})$ is the linear combination

$$
\Phi(\lambda, s, \boldsymbol{t}, \overline{\boldsymbol{t}})=\sum_{k=1}^{\infty} t_{k} \Phi_{k}(\lambda, s)+\sum_{k=1}^{\infty} \bar{t}_{k} \Phi_{-k}(\lambda, s) .
$$

$\Phi_{-k}(s, \lambda)$ 's are defined by the same formula as (2.6) with $k$ replaced by $-k$.

\subsection{Fermionic formulation}

We use the same formulation of 2D complex free fermions as in the previous work $[1,2]$. The Fourier modes $\psi_{n}, \psi_{n}^{*}, n \in \mathbb{Z}$ of the fermion fields

$$
\psi(z)=\sum_{n \in \mathbb{Z}} \psi_{n} z^{-n-1}, \quad \psi^{*}(z)=\sum_{n \in \mathbb{Z}} \psi_{n}^{*} z^{-n} .
$$

satisfy the anti-commutation relations

$$
\psi_{m} \psi_{n}^{*}+\psi_{n}^{*} \psi_{m}=\delta_{m+n, 0}, \quad \psi_{m} \psi_{n}+\psi_{n} \psi_{m}=0, \quad \psi_{m}^{*} \psi_{n}^{*}+\psi_{n}^{*} \psi_{m}^{*}=0 .
$$

The Fock spaces are decomposed to charge- $s$ sectors $(s \in \mathbb{Z})$ with the ground states

$$
\left\langle s\left|=\left\langle-\infty\left|\cdots \psi_{s-1}^{*} \psi_{s}^{*}, \quad\right| s\right\rangle=\psi_{-s} \psi_{-s+1} \cdots\right|-\infty\right\rangle
$$

and the excited states

$$
\left\langle s, \lambda\left|=\left\langle-\infty\left|\cdots \psi_{\lambda_{2}+s-1}^{*} \psi_{\lambda_{1}+s}^{*}, \quad\right| s, \lambda\right\rangle=\psi_{-\lambda_{1}-s} \psi_{-\lambda_{2}-s+1} \cdots\right|-\infty\right\rangle
$$

labelled by partitions.

Let us introduce the fermion bilinears

$$
\begin{aligned}
& L_{0}=\sum_{n \in \mathbb{Z}} n: \psi_{-n} \psi_{n}^{*}:, \quad W_{0}=\sum_{n \in \mathbb{Z}} n^{2}: \psi_{-n} \psi_{n}^{*}:, \\
& H_{k}=\sum_{n \in \mathbb{Z}} q^{k n}: \psi_{-n} \psi_{n}^{*}:, \quad J_{k}=\sum_{n \in \mathbb{Z}}: \psi_{-n} \psi_{n+k}^{*}:
\end{aligned}
$$

and the vertex operators $[9,14]$

$$
\Gamma_{ \pm}(z)=\exp \left(\sum_{k=1}^{\infty} \frac{z^{k}}{k} J_{ \pm k}\right), \quad \Gamma_{ \pm}^{\prime}(z)=\exp \left(-\sum_{k=1}^{\infty} \frac{(-z)^{k}}{k} J_{ \pm k}\right) .
$$

It is convenient to extend the definition of the vertex operators to multivariables $\boldsymbol{x}=\left(x_{1}, x_{2}, \ldots\right)$ :

$$
\Gamma_{ \pm}(\boldsymbol{x})=\prod_{i \geq 1} \Gamma_{ \pm}\left(x_{i}\right), \quad \Gamma_{ \pm}^{\prime}(\boldsymbol{x})=\prod_{i \geq 1} \Gamma_{ \pm}^{\prime}\left(x_{i}\right) .
$$


The matrix elements of these multi-variable vertex operators with respect to the states $\langle\lambda, r|,| \mu, s\rangle$ are skew Schur functions [12]:

$$
\begin{gathered}
\left\langle\lambda, r\left|\Gamma_{-}(\boldsymbol{x})\right| \mu, s\right\rangle=\left\langle\mu, s\left|\Gamma_{+}(\boldsymbol{x})\right| \lambda, r\right\rangle=\delta_{r s} s_{\lambda / \mu}(\boldsymbol{x}), \\
\left\langle\lambda, r\left|\Gamma_{-}^{\prime}(\boldsymbol{x})\right| \mu, s\right\rangle=\left\langle\mu, s\left|\Gamma_{+}^{\prime}(\boldsymbol{x})\right| \lambda, r\right\rangle=\delta_{r s}{ }^{{ }{ }{ }_{\lambda} /{ }^{\mathrm{t}} \mu}(\boldsymbol{x}) .
\end{gathered}
$$

Thus the special values of the Schur functions in the definition (2.5) and (2.12) of the deformed partition functions can be expressed as matrix elements of the vertex operators specialized to $\boldsymbol{x}=q^{-\rho}$. Since the potentials in the partition functions, too, can be expressed as

$$
|\lambda|+s(s+1)=\left\langle\lambda, s\left|L_{0}\right| \lambda, s\right\rangle, \quad \Phi_{k}(\lambda, s)=\left\langle\lambda, s\left|H_{k}\right| \lambda, s\right\rangle
$$

and the other matrix elements of $L_{0}$ and $H_{k}$ vanish, one can rewrite the partition functions as

$$
Z(s, \boldsymbol{t})=\left\langle s\left|\Gamma_{+}\left(q^{-\rho}\right) Q^{L_{0}} e^{H(\boldsymbol{t})} \Gamma_{-}\left(q^{-\rho}\right)\right| s\right\rangle
$$

and

$$
Z^{\prime}(s, \boldsymbol{t}, \overline{\boldsymbol{t}})=\left\langle s\left|\Gamma_{+}\left(q^{-\rho}\right) Q^{L_{0}} e^{H(\boldsymbol{t}, \overline{\boldsymbol{t}})} \Gamma_{-}^{\prime}\left(q^{-\rho}\right)\right| s\right\rangle,
$$

where

$$
H(\boldsymbol{t})=\sum_{k=1}^{\infty} t_{k} H_{k}, \quad H(\boldsymbol{t}, \overline{\boldsymbol{t}})=\sum_{k=1}^{\infty} t_{k} H_{k}+\sum_{k=1}^{\infty} \bar{t}_{k} H_{-k} .
$$

\section{Shift symmetries in fermionic formulation}

\subsection{Three sets of shift symmetries}

Let us introduce another set of fermion bilinears $\left\{V_{m}^{(k)}\right\}_{k, m \in \mathbb{Z}}$ :

$$
V_{m}^{(k)}=q^{k / 2} \oint_{|z|=R} \frac{d z}{2 \pi i} z^{m}: \psi\left(q^{k / 2} z\right) \psi^{*}\left(q^{-k / 2} z\right):=q^{-k m / 2} \sum_{n \in \mathbb{Z}} q^{k n}: \psi_{m-n} \psi_{n}^{*}:
$$

The radius $R>0$ is chosen arbitrarily. $H_{k}$ and $J_{k}$ are particular cases of these fermion bilinears:

$$
H_{k}=V_{0}^{(k)}, \quad J_{m}=V_{m}^{(0)} .
$$

$V_{m}^{(k)}$ 's satisfy the commutation relations

$$
\left[V_{m}^{(k)}, V_{n}^{(l)}\right]=\left(q^{(l m-k n) / 2}-q^{(k n-l m) / 2}\right)\left(V_{m+n}^{(k+l)}-\delta_{m+n, 0} \frac{q^{k+l}}{1-q^{k+l}}\right)
$$


for $k$ and $l$ with $k+l \neq 0$ and

$$
\left[V_{m}^{(k)}, V_{n}^{(-k)}\right]=\left(q^{-k(m+n)}-q^{k(m+n)}\right) V_{m+n}^{(0)}+m \delta_{m+n, 0},
$$

thus give a realization of (a Central extension of) the $2 \mathrm{D}$ quantum torus Lie algebra

"Shift symmetries" connect these fermion bilinears. There are three sets of fundamental shift symmetries:

\section{Theorem 1.}

(i) For $k>0$ and $m \in \mathbb{Z}$,

$$
\begin{aligned}
& \Gamma_{+}\left(q^{-\rho}\right)\left(V_{m}^{(k)}-\frac{q^{k}}{1-q^{k}} \delta_{m, 0}\right) \Gamma_{+}\left(q^{-\rho}\right)^{-1} \\
& =(-1)^{k} \Gamma_{-}\left(q^{-\rho}\right)^{-1}\left(V_{m+k}^{(k)}-\frac{q^{k}}{1-q^{k}} \delta_{m+k, 0}\right) \Gamma_{-}\left(q^{-\rho}\right) .
\end{aligned}
$$

(ii) For $k>0$ and $m \in \mathbb{Z}$,

$$
\begin{aligned}
& \Gamma_{+}^{\prime}\left(q^{-\rho}\right)\left(V_{m}^{(-k)}+\frac{1}{1-q^{k}} \delta_{m, 0}\right) \Gamma_{+}^{\prime}\left(q^{-\rho}\right)^{-1} \\
& =\Gamma_{-}^{\prime}\left(q^{-\rho}\right)^{-1}\left(V_{m+k}^{(-k)}+\frac{1}{1-q^{k}} \delta_{m+k, 0}\right) \Gamma_{-}^{\prime}\left(q^{-\rho}\right) .
\end{aligned}
$$

(iii) For $k, m \in \mathbb{Z}$,

$$
q^{W_{0} / 2} V_{m}^{(k)} q^{-W_{0} / 2}=V_{m}^{(k-m)}
$$

(3.3) and (3.5) are presented in our previous work [1, 2]. (3.4) is new, and we show the derivation of these shift symmetries in detail below. We shall also see why (3.3) and (3.4) do not hold for $k<0$.

Remark 1. In our previous work [1, 2], shift symmetries are presented in such a form as

$$
\begin{aligned}
& \Gamma_{-}\left(q^{-\rho}\right) \Gamma_{+}\left(q^{-\rho}\right)\left(V_{m}^{(k)}-\frac{q^{k}}{1-q^{k}} \delta_{m, 0}\right) \Gamma_{+}\left(q^{-\rho}\right)^{-1} \Gamma_{-}\left(q^{-\rho}\right)^{-1} \\
& =(-1)^{k}\left(V_{m+k}^{(k)}-\frac{q^{k}}{1-q^{k}} \delta_{m+k, 0}\right)
\end{aligned}
$$

and

$$
\begin{aligned}
& \Gamma_{-}^{\prime}\left(q^{-\rho}\right) \Gamma_{+}^{\prime}\left(q^{-\rho}\right)\left(V_{m}^{(-k)}+\frac{1}{1-q^{k}} \delta_{m, 0}\right) \Gamma_{+}^{\prime}\left(q^{-\rho}\right)^{-1} \Gamma_{-}^{\prime}\left(q^{-\rho}\right)^{-1} \\
& =V_{m+k}^{(-k)}+\frac{1}{1-q^{k}} \delta_{m+k, 0} .
\end{aligned}
$$


In this paper, however, we dare to avoid (3.6) and (3.7) because, firstly, it is (3.3) and (3.4) that are used in the subsequent consideration and, secondly, the operator products $\Gamma_{-}\left(q^{-\rho}\right) \Gamma_{+}\left(q^{-\rho}\right)$ and $\Gamma_{-}^{\prime}\left(q^{-\rho}\right) \Gamma_{+}^{\prime}\left(q^{-\rho}\right)$ in (3.6) and (3.7) require a careful treatment.

\subsection{Derivation of shift symmetries}

Derivation of (3.4) is parallel to the proof of $(3.3)[1,2]$. We start from the equality

$$
: \psi(z) \psi^{*}(w):=\psi(z) \psi^{*}(w)-\frac{1}{z-w}
$$

that holds for $|z|>|w|$. Since $|q|<1$, this equality can be specialized to

$$
: \psi\left(q^{-k / 2} z\right) \psi^{*}\left(q^{k / 2} z\right):=\psi\left(q^{-k / 2} z\right) \psi^{*}\left(q^{k / 2} z\right)-\frac{q^{k / 2}}{\left(1-q^{k}\right) z} .
$$

The fermion bilinears $V_{m}^{(-k)}$ in (3.4) can be thereby expressed as

$$
V_{m}^{(-k)}=q^{-k / 2} \oint_{|z|=R} \frac{d z}{2 \pi i} z^{m} \psi\left(q^{-k / 2} z\right) \psi^{*}\left(q^{k / 2} z\right)-\frac{1}{1-q^{k}} \delta_{m, 0} .
$$

We now use the formulae

$$
\begin{gathered}
\Gamma_{ \pm}^{\prime}(x) \psi(z) \Gamma_{ \pm}^{\prime}(x)^{-1}=\left(1+x z^{ \pm 1}\right) \psi(z), \\
\Gamma_{ \pm}^{\prime}(x) \psi^{*}(z) \Gamma_{ \pm}^{\prime}(x)^{-1}=\left(1+x z^{ \pm 1}\right)^{-1} \psi^{*}(z)
\end{gathered}
$$

that holds when $\left|x z^{ \pm 1}\right|<1$. The formulae for $\Gamma_{+}^{\prime}(x)$ in (3.9) imply that the equality

$$
\begin{aligned}
& \Gamma_{+}^{\prime}\left(q^{-\rho}\right) \psi\left(q^{-k / 2} z\right) \psi^{*}\left(q^{k / 2} z\right) \Gamma_{+}^{\prime}\left(q^{-\rho}\right)^{-1} \\
& =\frac{\prod_{i=1}^{\infty}\left(1+q^{i-1 / 2} \cdot q^{-k / 2} z\right)}{\prod_{i=1}^{\infty}\left(1+q^{i-1 / 2} \cdot q^{k / 2} z\right)} \psi\left(q^{-k / 2} z\right) \psi^{*}\left(q^{k / 2} z\right) \\
& =\prod_{i=1}^{k}\left(1+q^{i-(k+1) / 2} z\right) \cdot \psi\left(q^{-k / 2} z\right) \psi^{*}\left(q^{k / 2} z\right)
\end{aligned}
$$

holds for $|z|<|q|^{(k-1) / 2}$. Multiplying this equality by $q^{-k / 2} z^{m} / 2 \pi i$ and integrating it along the circle $|z|=R_{1}<|q|^{(k-1) / 2}$, we find that

$$
\begin{aligned}
& \Gamma_{+}^{\prime}\left(q^{-\rho}\right)\left(V_{m}^{(-k)}+\frac{1}{1-q^{k}} \delta_{m, 0}\right) \Gamma_{+}^{\prime}\left(q^{-\rho}\right)^{-1} \\
& =q^{-k / 2} \oint_{|z|=R_{1}} \frac{d z}{2 \pi i} z^{m} \prod_{i=1}^{k}\left(1+q^{i-(k+1) / 2} z\right) \cdot \psi\left(q^{-k / 2} z\right) \psi^{*}\left(q^{k / 2} z\right) .
\end{aligned}
$$


On the other hand, the formulae for $\Gamma_{-}^{\prime}(x)$ in (3.9) imply that the equality

$$
\begin{aligned}
& \Gamma_{-}^{\prime}\left(q^{-\rho}\right)^{-1} \psi\left(q^{-k / 2} z\right) \psi^{*}\left(q^{k / 2} z\right) \Gamma_{-}^{\prime}\left(q^{-\rho}\right) \\
& =\frac{\prod_{i=1}^{\infty}\left(1+q^{i-1 / 2} \cdot q^{-k / 2} z^{-1}\right)}{\prod_{i=1}^{\infty}\left(1+q^{i-1 / 2} \cdot q^{k / 2} z^{-1}\right)} \psi\left(q^{-k / 2} z\right) \psi^{*}\left(q^{k / 2} z\right) \\
& =\prod_{i=1}^{k}\left(1+q^{i-(k+1) / 2} z^{-1}\right) \cdot \psi\left(q^{-k / 2} z\right) \psi^{*}\left(q^{k / 2} z\right)
\end{aligned}
$$

holds for $|z|>|q|^{(k-1) / 2}$. Note here that the prefactors of $\psi\left(q^{-k / 2} z\right) \psi^{*}\left(q^{k / 2} z\right)$ in this equality and the previous ones are connected by the simple relation

$$
z^{m} \prod_{i=1}^{k}\left(1+q^{i-(k+1) / 2} z\right)=z^{m+k} \prod_{i=1}^{k}\left(1+q^{i-(k+1) / 2} z^{-1}\right) .
$$

Therefore, multiplying this relation by $q^{-k / 2} z^{m+k}$ and integrating it along the circle $|z|=R_{2}>|q|^{(k-1) / 2}$, we obtain the equality

$$
\begin{aligned}
& \Gamma_{-}\left(q^{-\rho}\right)^{-1}\left(V_{m+k}^{(-k)}+\frac{1}{1-q^{k}} \delta_{m+k, 0}\right) \Gamma_{-}^{\prime}\left(q^{-\rho}\right) \\
& =q^{-k / 2} \oint_{|z|=R_{2}} \frac{d z}{2 \pi i} z^{m} \prod_{i=1}^{k}\left(1+q^{i-(k+1) / 2} z\right) \cdot \psi\left(q^{-k / 2} z\right) \psi^{*}\left(q^{k / 2} z\right) .
\end{aligned}
$$

The right hand side of (3.10) and (3.12) are almost the same except for the difference of the contours. Actually they coincide, because the integrands have no singularity in the annulus $R_{1} \leq|z| \leq R_{2}$, and the contour can be continuously deformed therein without changing the contour integral. This completes the proof of (3.4).

\subsection{Where shift symmetries break down}

Let us consider what occurs when $k<0$, in other words, when $k$ is replaced by $-k$ in the setting of the proof of (3.4). We start from the equality

$$
: \psi(z) \psi^{*}(w):=-\psi^{*}(w) \psi(z)+\frac{1}{w-z}
$$

that holds for $|w|>|z|$. The fermion bilinear $V_{m}^{(k)}$ can be thereby expressed as

$$
V_{m}^{(k)}=-q^{k / 2} \oint_{|z|=R} \frac{d z}{2 \pi i} z^{m} \psi^{*}\left(q^{-k / 2} z\right) \psi\left(q^{k / 2} z\right)+\frac{q^{k}}{1-q^{k}} \delta_{m, 0}
$$


Under the adjoint action of $\Gamma_{ \pm}^{\prime}\left(q^{-\rho}\right)$, the operator product $\psi^{*}\left(q^{-k / 2} z\right) \psi\left(q^{k / 2} z\right)$ transforms as

$$
\begin{aligned}
& \Gamma_{+}^{\prime}\left(q^{-\rho}\right) \psi^{*}\left(q^{-k / 2} z\right) \psi\left(q^{k / 2} z\right) \Gamma_{+}^{\prime}\left(q^{-\rho}\right)^{-1} \\
& =\prod_{i=1}^{k}\left(1+q^{i-(k+1) / 2} z\right)^{-1} \cdot \psi^{*}\left(q^{-k / 2} z\right) \psi\left(q^{k / 2} z\right)
\end{aligned}
$$

and

$$
\begin{aligned}
& \Gamma_{-}^{\prime}\left(q^{-\rho}\right)^{-1} \psi^{*}\left(q^{-k / 2} z\right) \psi\left(q^{k / 2} z\right) \Gamma_{-}^{\prime}\left(q^{-\rho}\right) \\
& =\prod_{i=1}^{k}\left(1+q^{i-(k+1) / 2} z^{-1}\right)^{-1} \cdot \psi^{*}\left(q^{-k / 2} z\right) \psi\left(q^{k / 2} z\right) .
\end{aligned}
$$

Consequently, we obtain the following analogues of (3.10) and (3.10):

$$
\begin{aligned}
& \Gamma_{+}^{\prime}\left(q^{-\rho}\right)\left(-V_{m}^{(k)}+\frac{q^{k}}{1-q^{k}} \delta_{m, 0}\right) \Gamma_{+}^{\prime}\left(q^{-\rho}\right)^{-1} \\
& =q^{k / 2} \oint_{|z|=R_{1}} \frac{d z}{2 \pi i} z^{m} \prod_{i=1}^{k}\left(1+q^{i-(k+1) / 2} z\right)^{-1} \cdot \psi^{*}\left(q^{-k / 2} z\right) \psi\left(q^{k / 2} z\right), \\
& \Gamma_{-}^{\prime}\left(q^{-\rho}\right)^{-1}\left(-V_{m-k}^{(k)}+\frac{q^{k}}{1-q^{k}} \delta_{m-k, 0}\right) \Gamma_{-}^{\prime}\left(q^{-\rho}\right) \\
& =q^{k / 2} \oint_{|z|=R_{2}} \frac{d z}{2 \pi i} z^{m} \prod_{i=1}^{k}\left(1+q^{i-(k+1) / 2} z\right)^{-1} \cdot \psi^{*}\left(q^{-k / 2} z\right) \psi\left(q^{k / 2} z\right) .
\end{aligned}
$$

Unlike the proof of (3.4), the integrands of these contour integrals have poles, one of which sits on the circle $|z|=|q|^{(k-1) / 2}$. The contours $|z|=R_{1}$ and $|z|=R_{2}$ are separated by this circle, and cannot be reached from the other by continuous deformation without crossing the poles. Thus an equality like (3.4) does not hold.

This explains why (3.4) breaks down for $k<0$. By the same reasoning, one can see that (3.3) does not hold for $k<0$. 


\section{Partition functions as tau functions}

\subsection{Previous model}

In our previous work $[1,2]$, the partition function $Z(s, \boldsymbol{t})$ is shown to be related to a tau function $\tau(x, \boldsymbol{t})$ of the 1D Toda hierarchy as

$$
Z(s, \boldsymbol{t})=\exp \left(\sum_{k=1}^{\infty} \frac{t_{k} q^{k}}{1-q^{k}}\right) q^{-s(s+1)(2 s+1) / 6} \tau(s, \iota(\boldsymbol{t})),
$$

where $\iota(\boldsymbol{t})$ denotes the alternating inversion

$$
\iota(\boldsymbol{t})=\left(-t_{1}, t_{2},-t_{3}, \ldots,(-1)^{k} t_{k}, \ldots\right)
$$

of $\boldsymbol{t}=\left(t_{1}, t_{2}, \ldots\right)$. The tau function $\tau(s, \boldsymbol{t})$ has the fermionic expression

$$
\tau(s, \boldsymbol{t})=\left\langle s\left|\exp \left(\sum_{k=1}^{\infty} t_{k} J_{k}\right) g\right| s\right\rangle,
$$

where

$$
g=q^{W_{0} / 2} \Gamma_{-}\left(q^{-\rho}\right) \Gamma_{+}\left(q^{-\rho}\right) Q^{L_{0}} \Gamma_{-}\left(q^{-\rho}\right) \Gamma_{+}\left(q^{-\rho}\right) q^{W_{0} / 2} .
$$

The two sets (3.3) and (3.5) of shift symmetries are used to derive (4.1) from the fermionic expression (2.15) of the partition function.

By the same same shift symmetries, one can also show that $g$ satisfies the algebraic relations

$$
J_{k} g=g J_{-k}, \quad k=1,2, \ldots
$$

This implies that the associated tau function [13]

$$
\tau(s, \boldsymbol{t}, \overline{\boldsymbol{t}})=\left\langle s\left|\exp \left(\sum_{k=1}^{\infty} t_{k} J_{k}\right) g \exp \left(-\sum_{k=1}^{\infty} \bar{t}_{k} J_{-k}\right)\right| s\right\rangle
$$

of the 2D Toda hierarchy depends on $\boldsymbol{t}$ and $\overline{\boldsymbol{t}}$ through their difference:

$$
\tau(s, \boldsymbol{t}, \overline{\boldsymbol{t}})=\tau(s, \boldsymbol{t}-\overline{\boldsymbol{t}}) .
$$

Consequently, $\tau(s, \boldsymbol{t})$ can be expressed in different forms, e.g.,

$$
\begin{aligned}
\tau(s, \boldsymbol{t}) & =\left\langle s\left|g \exp \left(\sum_{k=1}^{\infty} t_{k} J_{-k}\right)\right| s\right\rangle \\
& =\left\langle s\left|\exp \left(\frac{1}{2} \sum_{k=1}^{\infty} t_{k} J_{k}\right) g\left(\frac{1}{2} \sum_{k=1}^{\infty} t_{k} J_{-k}\right)\right| s\right\rangle .
\end{aligned}
$$




\subsection{Modified model}

We now turn to the modified model, and derive an analogue of (4.1) from the fermionic expression (2.16). The derivation is mostly parallel to the case of the previous model $[1,2]$.

First step: The first set (3.3) of shift symmetries, applied to $H_{k}=V_{0}^{(k)}$, $k=1,2, \ldots$, imply that

$$
\Gamma_{+}\left(q^{-\rho}\right) H_{k} \Gamma_{+}\left(q^{-\rho}\right)^{-1}-\frac{q^{k}}{1-q^{k}}=(-1)^{k} \Gamma_{-}\left(q^{-\rho}\right)^{-1} V_{k}^{(k)} \Gamma_{-}\left(q^{-\rho}\right) .
$$

Moreover, the third set (3.5) of shift symmetries imply that

$$
V_{k}^{(k)}=q^{-W_{0} / 2} J_{k} q^{W_{0} / 2} .
$$

From these equalities, we obtain the algebraic relation

$$
\Gamma_{+}\left(q^{-\rho}\right) H_{k} \Gamma_{+}\left(q^{-\rho}\right)^{-1}=(-1)^{k} \Gamma_{-}\left(q^{-\rho}\right)^{-1} q^{-W_{0} / 2} J_{k} q^{W_{0} / 2} \Gamma_{-}\left(q^{-\rho}\right)+\frac{q^{k}}{1-q^{k}}
$$

that connects $H_{k}$ and $J_{k}$. This algebraic relation can be exponentiated as

$$
\begin{aligned}
& \Gamma_{+}\left(q^{-\rho}\right) \exp \left(\sum_{k=1}^{\infty} t_{k} H_{k}\right) \Gamma_{+}\left(q^{-\rho}\right)^{-1}=\exp \left(\sum_{k=1}^{\infty} \frac{q^{k} t_{k}}{1-q^{k}}\right) \times \\
& \times \Gamma_{-}\left(q^{-\rho}\right)^{-1} q^{-W_{0} / 2} \exp \left(\sum_{k=1}^{\infty}(-1)^{k} t_{k} J_{k}\right) q^{W_{0} / 2} \Gamma_{-}\left(q^{-\rho}\right) .
\end{aligned}
$$

Second step: The second set (3.4) of shift symmetries, applied to $H_{-k}=$ $V_{0}^{(-k)}, k=1,2, \ldots$, imply that

$$
\Gamma_{-}^{\prime}\left(q^{-\rho}\right)^{-1} H_{-k} \Gamma_{-}^{\prime}\left(q^{-\rho}\right)+\frac{1}{1-q^{k}}=\Gamma_{+}^{\prime}\left(q^{-\rho}\right) V_{-k}^{(-k)} \Gamma_{+}^{\prime}\left(q^{-\rho}\right)^{-1} .
$$

Moreover, the third set (3.5) of shift symmetries imply that

$$
V_{-k}^{(-k)}=q^{-W_{0} / 2} J_{-k} q^{W_{0} / 2} .
$$

Consequently, we obtain the algebraic relation

$$
\Gamma_{-}^{\prime}\left(q^{-\rho}\right)^{-1} H_{-k} \Gamma_{-}^{\prime}\left(q^{-\rho}\right)=\Gamma_{+}^{\prime}\left(q^{-\rho}\right) q^{-W_{0} / 2} J_{-k} q^{W_{0} / 2} \Gamma_{+}^{\prime}\left(q^{-\rho}\right)^{-1}-\frac{1}{1-q^{k}}
$$


which can be exponentiated as

$$
\begin{aligned}
& \Gamma_{-}^{\prime}\left(q^{-\rho}\right)^{-1} \exp \left(\sum_{k=1}^{\infty} \bar{t}_{k} H_{-k}\right) \Gamma_{-}^{\prime}\left(q^{-\rho}\right)=\exp \left(-\sum_{k=1}^{\infty} \frac{\bar{t}_{k}}{1-q^{k}}\right) \times \\
& \times \Gamma_{+}^{\prime}\left(q^{-\rho}\right) q^{-W_{0} / 2} \exp \left(\sum_{k=1}^{\infty} \bar{t}_{k} J_{-k}\right) q^{W_{0} / 2} \Gamma_{+}^{\prime}\left(q^{-\rho}\right)^{-1} .
\end{aligned}
$$

Third step: Let us factorize the operator in (2.16) as

$$
\begin{aligned}
& \Gamma_{+}\left(q^{-\rho}\right) Q^{L_{0}} e^{H(\boldsymbol{t}, \bar{t})} \Gamma_{-}^{\prime}\left(q^{-\rho}\right)=\Gamma_{+}\left(q^{-\rho}\right) \exp \left(\sum_{k=1}^{\infty} t_{k} H_{k}\right) \Gamma_{+}\left(q^{-\rho}\right)^{-1} \times \\
& \times \Gamma_{+}\left(q^{-\rho}\right) Q^{L_{0}} \Gamma_{-}^{\prime}\left(q^{-\rho}\right) \Gamma_{-}^{\prime}\left(q^{-\rho}\right)^{-1} \exp \left(\sum_{k=1}^{\infty} \bar{t}_{k} H_{-k}\right) \Gamma_{-}^{\prime}\left(q^{-\rho}\right) .
\end{aligned}
$$

By (4.6) and (4.7), we can rewrite it as

$$
\begin{aligned}
& \Gamma_{+}\left(q^{-\rho}\right) Q^{L_{0}} e^{H(\boldsymbol{t}, \overline{\boldsymbol{t}})} \Gamma_{-}^{\prime}\left(q^{-\rho}\right)=\exp \left(\sum_{k=1}^{\infty} \frac{q^{k} t_{k}-\bar{t}_{k}}{1-q^{k}}\right) \Gamma_{-}\left(q^{-\rho}\right)^{-1} q^{-W_{0} / 2} \times \\
& \times \exp \left(\sum_{k=1}^{\infty}(-1)^{k} t_{k} J_{k}\right) g^{\prime} \exp \left(\sum_{k=1}^{\infty} \bar{t}_{k} J_{-k}\right) q^{W_{0} / 2} \Gamma_{+}^{\prime}\left(q^{-\rho}\right)^{-1},
\end{aligned}
$$

where

$$
g^{\prime}=q^{W_{0} / 2} \Gamma_{-}\left(q^{-\rho}\right) \Gamma_{+}\left(q^{-\rho}\right) Q^{L_{0}} \Gamma_{-}^{\prime}\left(q^{-\rho}\right) \Gamma_{+}^{\prime}\left(q^{-\rho}\right) q^{-W_{0} / 2} .
$$

Plugging this expression into (2.16) and noting that

$$
\begin{aligned}
\langle s| \Gamma_{-}\left(q^{-\rho}\right) q^{-W_{0} / 2} & =q^{-s(s+1)(2 s+1) / 12}\langle s|, \\
q^{W_{0} / 2} \Gamma_{+}^{\prime}\left(q^{-\rho}\right)|s\rangle & =|s\rangle q^{s(s+1)(2 s+1) / 12},
\end{aligned}
$$

we end up with the following result:

Theorem 2. $Z^{\prime}(s, \boldsymbol{t}, \overline{\boldsymbol{t}})$ can be expressed as

$$
Z^{\prime}(s, \boldsymbol{t}, \overline{\boldsymbol{t}})=\exp \left(\sum_{k=1}^{\infty} \frac{q^{k} t_{k}-\bar{t}_{k}}{1-q^{k}}\right) \tau^{\prime}(s, \iota(\boldsymbol{t}),-\overline{\boldsymbol{t}}),
$$

where $\tau^{\prime}(s, \boldsymbol{t}, \overline{\boldsymbol{t}})$ is the tau function

$$
\tau^{\prime}(s, \boldsymbol{t}, \overline{\boldsymbol{t}})=\left\langle s\left|\exp \left(\sum_{k=1}^{\infty} t_{k} J_{k}\right) g^{\prime} \exp \left(-\sum_{k=1}^{\infty} \bar{t}_{k} J_{-k}\right)\right| s\right\rangle
$$

of the 2D Toda hierarchy defined by the operator (4.8). 
Remark 2. If the shift symmetries (3.3) and (3.4) were valid for $k<0$ as well, one could show that $g^{\prime}$ satisfy the algebraic relations

$$
J_{ \pm k} g^{\prime}=g^{\prime} J_{ \pm k}, \quad k=1,2, \ldots \quad \text { (wrong statement) }
$$

This imply that the tau function would become an almost trivial one:

$$
\tau^{\prime}(s, \boldsymbol{t}, \overline{\boldsymbol{t}})=\exp \left(-\sum_{k=1}^{\infty} k t_{k} \bar{t}_{k}\right)\left\langle s\left|g^{\prime}\right| s\right\rangle \quad \text { (wrong statement) }
$$

This is a typical example of wrong consequences derived by a careless use of shift symmetries [5].

\section{$5 \quad$ Shift symmetries in matrix formulation}

\subsection{Matrix representation of fermion bilinears}

It is well known [12] that the mapping

$$
X=\sum_{i, j \in \mathbb{Z}} x_{i j} E_{i j} \mapsto \hat{X}=\sum_{i, j \in \mathbb{Z}} x_{i j}: \psi_{-i} \psi_{j}^{*}:
$$

gives a projective representation (in other words, a central extension) of the Lie algebra $\mathrm{gl}(\infty)$ of $\mathbb{Z} \times \mathbb{Z}$ matrices. In the following, we naively identify $\hat{X}$ and $X$. The fundamental fermion bilinears $L_{0}, W_{0}, H_{k}, J_{k}$ can be thereby identified with the $\mathbb{Z} \times \mathbb{Z}$ matrices

$$
L_{0}=\Delta, \quad W_{0}=\Delta^{2}, \quad H_{k}=q^{k \Delta}, \quad J_{k}=\Lambda^{k},
$$

where $\Delta$ and $\Lambda$ are the matrices

$$
\Delta=\sum_{i \in \mathbb{Z}} i E_{i i}, \quad \Lambda=\sum_{i \in \mathbb{Z}} E_{i, i+1}
$$

that satisfy the twisted canonical commutation relation

$$
[\Lambda, \Delta]=\Lambda
$$

$\Lambda$ and $\Delta$ amount to the shift and multiplication operators $e^{\partial / \partial s}$ and $s$ in the Lax formalism of the 2D Toda hierarchy [10, 11],

The fermionic realization $\left\{V_{m}^{(k)}\right\}_{k, m \in \mathbb{Z}}$ of the quantum torus Lie algebra correspond to the $\mathbb{Z} \times \mathbb{Z}$ matrices

$$
V_{m}^{(k)}=q^{-k m / 2} \Lambda^{m} q^{k \Delta}=q^{k m / 2} q^{k \Delta} \Lambda^{m} .
$$


These matrices form an associative algebra rather than a Lie algebra. The commutation relations

$$
\left[V_{m}^{(k)}, V_{n}^{(l)}\right]=\left(q^{(l m-k n) / 2}-q^{(k n-l m) / 2}\right) V_{m+n}^{(k+l)}
$$

can be derived from the fundamental relation

$$
\Lambda q^{\Delta}=q q^{\Delta} \Lambda
$$

of the generators $\Lambda$ and $q^{\Delta}$.

Last but not least, the vertex operators $\Gamma_{ \pm}(x)$ and $\Gamma_{ \pm}^{\prime}(x)$ turn out to have a matrix representation of the form

$$
\begin{gathered}
\Gamma_{ \pm}(x)=\exp \left(\sum_{k=1}^{\infty} \frac{x^{k}}{k} \Lambda^{ \pm k}\right)=\left(1-x \Lambda^{ \pm 1}\right)^{-1}, \\
\Gamma_{ \pm}^{\prime}(x)=\exp \left(-\sum_{k=1}^{\infty} \frac{(-x)^{k}}{k} \Lambda^{ \pm}\right)=\left(1+x \Lambda^{ \pm 1}\right) .
\end{gathered}
$$

The inverse of $1-x \Lambda^{ \pm 1}$ is understood to be the geometric series

$$
\left(1-x \Lambda^{ \pm 1}\right)^{-1}=1+x \Lambda^{ \pm 1}+x^{2} \Lambda^{ \pm 2}+\cdots .
$$

Among the four matrices of (5.7), those with label "+" are upper triangular, and those with label "-" are lower triangular. The matrix representation of $\Gamma_{ \pm}\left(q^{-\rho}\right)$ and $\Gamma_{ \pm}^{\prime}\left(q^{-\rho}\right)$ is an infinite product of these matrices specialized to $x=q^{i-1 / 1}$ :

$$
\begin{gathered}
\Gamma_{ \pm}\left(q^{-\rho}\right)=\prod_{i=1}^{\infty}\left(1-q^{i-1 / 2} \Lambda^{ \pm 1}\right)^{-1} \\
\Gamma_{ \pm}^{\prime}\left(q^{-\rho}\right)=\prod_{i=1}^{\infty}\left(1+q^{i-1 / 2} \Lambda^{ \pm 1}\right) .
\end{gathered}
$$

Thus $\Gamma_{ \pm}\left(q^{-\rho}\right)$ and $\Gamma_{ \pm}^{\prime}\left(q^{-\rho}\right)$ may be thought of as matrix-valued quantum dilogarithmic functions in the sense of Faddeev et al. [29, 30].

\subsection{Reformulation of shift symmetries}

The shift symmetries (3.3), (3.4) and (3.5) can be reformulated in the matrix representation as follows. 
Theorem 3. The $\mathbb{Z} \times \mathbb{Z}$ matrices (5.2), (5.4) and (5.8) satisfy the following analogues of (3.3), (3.4) and (3.5):

$$
\begin{gathered}
\Gamma_{+}\left(q^{-\rho}\right) V_{m}^{(k)} \Gamma_{+}\left(q^{-\rho}\right)^{-1}=(-1)^{k} \Gamma_{-}\left(q^{-\rho}\right)^{-1} V_{m+k}^{(k)} \Gamma_{-}\left(q^{-\rho}\right) \\
\Gamma_{+}^{\prime}\left(q^{-\rho}\right) V_{m}^{(-k)} \Gamma_{+}^{\prime}\left(q^{-\rho}\right)^{-1}=\Gamma_{-}^{\prime}\left(q^{-\rho}\right)^{-1} V_{m+k}^{(-k)} \Gamma_{-}^{\prime}\left(q^{-\rho}\right) \\
q^{\Delta^{2} / 2} V_{m}^{(k)} q^{-\Delta^{2} / 2}=V_{m}^{(k-m)}
\end{gathered}
$$

Here $k$ and $m$ in (5.9) and (5.10) range over $k>0$ and $m \in \mathbb{Z}$, and (5.11) holds for $k \in \mathbb{Z}$ and $m \in \mathbb{Z}$.

Proof. For a comparison with the proof of (3.4), let us show the derivation of (5.10) in detail. A technical clue is the algebraic relation

$$
q^{-k \Delta} \Lambda^{n} q^{k \Delta}=q^{k n} \Lambda^{n}
$$

that is a partially exponentiated form of (5.3).

We first consider the left hand side of (5.10), namely,

$$
\Gamma_{+}^{\prime}\left(q^{-\rho}\right) V_{m}^{(-k)} \Gamma_{+}^{\prime}\left(q^{-\rho}\right)^{-1}=q^{k m / 2} \Lambda^{m} \Gamma_{+}^{\prime}\left(q^{-\rho}\right) q^{-k \Delta} \Gamma_{+}^{\prime}\left(q^{-\rho}\right)^{-1} .
$$

(5.12) implies that

$q^{-k \Delta} \Gamma_{+}^{\prime}\left(q^{-\rho}\right)^{-1} q^{k \Delta}=\prod_{i=1}^{\infty}\left(1+q^{i-1 / 2} q^{-k \Delta} \Lambda q^{k \Delta}\right)^{-1}=\prod_{i=1}^{\infty}\left(1+q^{i+k-1 / 2} \Lambda\right)^{-1}$.

Multiplying this equality by $\Gamma_{+}^{\prime}\left(q^{-\rho}\right)$ and $q^{-k \Delta}$ from the left and right sides yields the equality

$$
\begin{aligned}
\Gamma_{+}^{\prime}\left(q^{-\rho}\right) q^{-k \Delta} \Gamma_{+}^{\prime}\left(q^{-\rho}\right)^{-1} & =\prod_{i=1}^{\infty}\left(1+q^{i-1 / 2} \Lambda\right) \cdot \prod_{i=1}^{\infty}\left(1+q^{i+k-1 / 2} \Lambda\right)^{-1} \cdot q^{-k \Delta} \\
& =\prod_{i=1}^{k}\left(1+q^{i-1 / 2} \Lambda\right) \cdot q^{-k \Delta}
\end{aligned}
$$

which implies that

$$
\Gamma_{+}^{\prime}\left(q^{-\rho}\right) V_{m}^{(-k)} \Gamma_{+}^{\prime}\left(q^{-\rho}\right)^{-1}=q^{k m / 2} \Lambda^{m} \prod_{i=1}^{k}\left(1+q^{i-1 / 2} \Lambda\right) \cdot q^{-k \Delta} .
$$

We now consider the right hand side of (5.10), namely,

$$
\Gamma_{-}^{\prime}\left(q^{-\rho}\right)^{-1} V_{m+k}^{(-k)} \Gamma_{-}^{\prime}\left(q^{-\rho}\right)=q^{k(m+k) / 2} \Lambda^{m+k} \Gamma_{-}^{\prime}\left(q^{-\rho}\right)^{-1} q^{-k \Delta} \Gamma_{-}^{\prime}\left(q^{-\rho}\right) .
$$


In the same way as we have followed above, we can derive the equality

$$
\begin{aligned}
\Gamma_{-}^{\prime}\left(q^{-\rho}\right)^{-1} q^{-k \Delta} \Gamma_{-}^{\prime}\left(q^{-\rho}\right) & =\prod_{i=1}^{\infty}\left(1+q^{i-1 / 2} \Lambda^{-1}\right)^{-1} \cdot \prod_{i=1}^{\infty}\left(1+q^{i-k-1 / 2} \Lambda^{-1}\right) \cdot q^{-k \Delta} \\
& =\prod_{i=1}^{k}\left(1+q^{i-k-1 / 2} \Lambda^{-1}\right) \cdot q^{-k \Delta} .
\end{aligned}
$$

Since

$$
q^{k^{2} / 2} \Lambda^{k} \prod_{i=1}^{k}\left(1+q^{i-k-1 / 2} \Lambda^{-1}\right)=\prod_{i=1}^{k}\left(1+q^{i-1 / 2} \Lambda\right),
$$

we can conclude that

$$
\Gamma_{-}^{\prime}\left(q^{-\rho}\right)^{-1} V_{m+k}^{(-k)} \Gamma_{-}^{\prime}\left(q^{-\rho}\right)=q^{k m / 2} \Lambda^{m} \prod_{i=1}^{k}\left(1+q^{i-1 / 2} \Lambda\right) \cdot q^{-k \Delta} .
$$

Thus both hand sides of (5.10) turn out to coincide. We can derive (5.9) in the same way from the equalities

$$
\begin{gathered}
\Gamma_{+}\left(q^{-\rho}\right) V_{m}^{(k)} \Gamma_{+}\left(q^{-\rho}\right)^{-1}=q^{-k m / 2} \Lambda^{m} \prod_{i=1}^{k}\left(1-q^{i-k+1 / 2} \Lambda\right) \cdot q^{k \Delta}, \\
\Gamma_{-}\left(q^{-\rho}\right)^{-1} V_{m+k}^{(k)} \Gamma_{-}\left(q^{-\rho}\right)=q^{-k(m+k) / 2} \Lambda^{m+k} \prod_{i=1}^{k}\left(1-q^{i-1 / 2} \Lambda^{-1}\right) \cdot q^{k \Delta}
\end{gathered}
$$

and

$$
q^{-k^{2} / 2} \Lambda^{k} \prod_{k=1}^{k}\left(1-q^{i-1 / 2} \Lambda^{-1}\right)=(-1)^{k} q^{-k m / 2} \Lambda^{m} \prod_{k=1}^{k}\left(1-q^{i-k+1 / 2} \Lambda\right) \cdot q^{k \Delta} .
$$

Lastly, (5.11) can be derived by straightforward calculation of the matrix elements of both hand sides as done in the language of fermions $[1,2]$.

\subsection{Where shift symmetries break down}

Just as in the fermionic formulation, (5.9) and (5.10) do not hold for $k<0$. Let us replace $k \rightarrow-k$ in (5.9) and (5.10), and show that the equalities do not hold.

By replacing $k \rightarrow-k$, both hand sides of (5.9) turn into

$$
\Gamma_{+}^{\prime}\left(q^{-\rho}\right) V_{m}^{(k)} \Gamma_{+}^{\prime}\left(q^{-\rho}\right)^{-1}=q^{-k m / 2} \Lambda^{m} \Gamma_{+}^{\prime}\left(q^{-\rho}\right) q^{k \Delta} \Gamma_{+}^{\prime}\left(q^{-\rho}\right)^{-1}
$$


and

$$
\Gamma_{-}^{\prime}\left(q^{-\rho}\right)^{-1} V_{m-k}^{(k)} \Gamma_{-}^{\prime}\left(q^{-\rho}\right)=q^{-k(m-k) / 2} \Lambda^{m-k} \Gamma_{-}^{\prime}\left(q^{-\rho}\right)^{-1} q^{k \Delta} \Gamma_{-}^{\prime}\left(q^{-\rho}\right) .
$$

As in the proof of Theorem 3, we can calculate the triple product on the right hand side as

$$
\Gamma_{+}^{\prime}\left(q^{-\rho}\right) V_{m}^{(k)} \Gamma_{+}^{\prime}\left(q^{-\rho}\right)^{-1}=q^{-k m / 2} \Lambda^{m} \prod_{i=1}^{k}\left(1+q^{i-k-1 / 2} \Lambda\right)^{-1} \cdot q^{k \Delta}
$$

and

$$
\Gamma_{-}^{\prime}\left(q^{-\rho}\right)^{-1} V_{m-k}^{(k)} \Gamma_{-}^{\prime}\left(q^{-\rho}\right)=q^{-k(m-k) / 2} \Lambda^{m-k} \prod_{i=1}^{k}\left(1+q^{i-1 / 2} \Lambda^{-1}\right)^{-1} \cdot q^{k \Delta} .
$$

Let us consider the inverse matrices $\left(1+q^{i-k-1 / 2} \Lambda\right)^{-1}$ and $\left(1+q^{i-1 / 2} \Lambda^{-1}\right)^{-1}$ that show up in these expressions. They are understood to be given by geometric series:

$$
\begin{aligned}
& \left(1+q^{i-k-1 / 2} \Lambda\right)^{-1}=1-q^{i-k-1 / 2} \Lambda+\left(q^{i-k-1 / 2} \Lambda\right)^{2}-\cdots, \\
& \left(1+q^{i-1 / 2} \Lambda^{-1}\right)^{-1}=1-q^{i-1 / 2} \Lambda^{-1}+\left(q^{i-1 / 2} \Lambda^{-1}\right)^{2}-\cdots .
\end{aligned}
$$

In particular, $\left(1+q^{i-k-1 / 2} \Lambda\right)^{-1}$ and $\left(1+q^{i-1 / 2} \Lambda^{-1}\right)^{-1}$ are upper triangular and lower triangular, respectively. Consequently, unlike the equality (5.13) that underlies (5.10), we have the inequality

$$
q^{k / 2} \Lambda^{-k} \prod_{i=1}^{k}\left(1+q^{i-1 / 2} \Lambda^{-1}\right)^{-1} \neq \prod_{i=1}^{k}\left(1+q^{i-k-1 / 2} \Lambda\right)^{-1} .
$$

(5.14) implies that

$$
\Gamma_{+}^{\prime}\left(q^{-\rho}\right) V_{m}^{(k)} \Gamma_{+}^{\prime}\left(q^{-\rho}\right)^{-1} \neq \Gamma_{-}^{\prime}\left(q^{-\rho}\right)^{-1} V_{m-k}^{(k)} \Gamma_{-}^{\prime}\left(q^{-\rho}\right) .
$$

By the same reasoning, one can confirm that

$$
\Gamma_{+}\left(q^{-\rho}\right) V_{m}^{(-k)} \Gamma_{+}\left(q^{-\rho}\right)^{-1} \neq(-1)^{k} \Gamma_{-}\left(q^{-\rho}\right)^{-1} V_{m-k}^{(-k)} \Gamma_{-}\left(q^{-\rho}\right) .
$$

\section{Relation to Ablowitz-Ladik hierarchy}

\subsection{Two reductions of $2 \mathrm{D}$ Toda hierarchy}

In the Lax formalism $[10,11]$, the $2 \mathrm{D}$ Toda hierarchy is formulated as a system of Lax equations

$$
\begin{array}{ll}
\frac{\partial L}{\partial t_{k}}=\left[B_{k}, L\right], & \frac{\partial \bar{L}}{\partial t_{k}}=\left[B_{k}, \bar{L}\right], \\
\frac{\partial L}{\partial \bar{t}_{k}}=\left[\bar{B}_{k}, L\right], & \frac{\partial \bar{L}}{\partial \bar{t}_{k}}=\left[\bar{B}_{k}, \bar{L}\right]
\end{array}
$$


for two Lax operators $L$ and $\bar{L}$. In the case of a bi-infinite lattice, $L$ and $\bar{L}$ are difference operators (i.e., linear combinations of the shift operators $e^{n \partial_{s}}$ in the lattice coordinate $s$ ) of the form

$$
L=e^{\partial_{s}}+\sum_{n=1}^{\infty} u_{n} e^{(1-n) \partial_{s}}, \quad \bar{L}^{-1}=\bar{u}_{0} e^{-\partial_{s}}+\sum_{n=1}^{\infty} \bar{u}_{n} e^{(n-1) \partial_{s}},
$$

where $u_{n}$ and $\bar{u}_{n}$ depend on $s, \boldsymbol{t}$ and $\overline{\boldsymbol{t}} . B_{k}$ and $\bar{B}_{k}$ are constructed from $L$ and $\bar{L}$ as

$$
B_{k}=\left(L^{k}\right)_{\geq 0}, \quad \bar{B}_{k}=\left(\bar{L}^{-k}\right)_{<0},
$$

where $(\quad)_{\geq 0}$ and $(\quad)_{<0}$ denote the projections to linear combinations of $e^{n \partial_{s}}$ for $n \geq 0$ and $n<0$.

The 1D Toda hierarchy and the Ablowitz-Ladik hierarchy are two typical reductions of the 2D Toda hierarchy. Let us recall the reduction procedure.

\section{Reduction to 1D Toda hierarchy}

The 1D Toda hierarchy can be derived from the 2D Toda hierarchy by imposing the reduction condition

$$
L=\bar{L}^{-1} .
$$

Both hand side of this equality define a difference operator of the form

$$
\mathfrak{L}=e^{\partial_{s}}+b+c e^{-\partial_{s}}
$$

that satisfies the Lax equations

$$
\frac{\partial \mathfrak{L}}{\partial t_{k}}=\left[B_{k}, \mathfrak{L}\right], \quad \frac{\partial \mathfrak{L}}{\partial \bar{t}_{k}}=\left[\bar{B}_{k}, \mathfrak{L}\right]
$$

Actually, since $B_{k}, \bar{B}_{k}$ and $\mathfrak{L}$ are related as

$$
B_{k}+\bar{B}_{k}=\mathfrak{L}^{k},
$$

the time evolutions in $\boldsymbol{t}$ and $\overline{\boldsymbol{t}}$ are no longer independent in the sense that

$$
\frac{\partial \mathfrak{L}}{\partial t_{k}}+\frac{\partial \mathfrak{L}}{\partial \bar{t}_{k}}=\left[B_{k}, \mathfrak{L}\right]+\left[\bar{B}_{k}, \mathfrak{L}\right]=0 .
$$

Thus the time evolutions in $\overline{\boldsymbol{t}}$ are redundant, and we are left with just one set of Lax equations

$$
\frac{\partial \mathfrak{L}}{\partial t_{k}}=\left[B_{k}, \mathfrak{L}\right], \quad B_{k}=\left(\mathfrak{L}^{k}\right)_{\geq 0} .
$$

This is one of the well known Lax forms of the 1D Toda hierarchy. 


\section{Reduction to Ablowitz-Ladik hierarchy}

The Ablowitz-Ladik hierarchy can be derived from the 2D Toda hierarchy as a kind of "rational reduction". In the formulation of Brini et al. [24], $L$ and $\bar{L}$ are chosen to be quotients

$$
L=B C^{-1}, \quad \bar{L}^{-1}=C B^{-1}
$$

of two difference operators of the form

$$
B=e^{\partial_{s}}-b, \quad C=1-c e^{-\partial_{s}} .
$$

$B^{-1}$ and $C^{-1}$ are understood to be difference operators of the form

$$
\begin{aligned}
& B^{-1}=-\sum_{k=0}^{\infty}\left(b^{-1} e^{\partial_{s}}\right)^{k} b^{-1}=-b(s)^{-1}-\sum_{k=1}^{\infty} b(s)^{-1} \cdots b(s+k)^{-1} e^{k \partial_{s}}, \\
& C^{-1}=1+\sum_{k=1}^{\infty}\left(c e^{-\partial_{s}}\right)^{k}=1+\sum_{k=1}^{\infty} c(s) c(s-1) \cdots c(s-k+1) e^{-k \partial_{s}},
\end{aligned}
$$

where $b(s)$ and $c(s)$ are abbreviations of $c(s, \boldsymbol{t}, \overline{\boldsymbol{t}})$ and $c(s, \boldsymbol{t}, \overline{\boldsymbol{t}}) . B$ and $C$ have inverses of different types,

$$
\begin{gathered}
B^{-1}=\sum_{k=0}^{\infty} e^{-\partial_{s}}\left(b e^{-\partial_{s}}\right)^{k}=e^{-\partial_{s}}+\sum_{k=1}^{\infty} b(s-1) \cdots b(s-k+1) e^{-k \partial_{s}}, \\
C^{-1}=-\sum_{k=1}^{\infty}\left(e^{\partial_{s}} c^{-1}\right)^{k}=-\sum_{k=1}^{\infty} c(s+1)^{-1} \cdots c(s+k)^{-1} e^{k \partial_{s}}
\end{gathered}
$$

but they are not used here. (6.6) imply that $\left(C B^{-1}\right)^{-1} \neq B C^{-1}$. Thus we can avoid the trivial case where $L=\bar{L}=e^{\partial_{s}}$. Under the reduction condition (6.5), the Lax equations (6.1) can be reduced to the following equations:

$$
\begin{aligned}
& \frac{\partial B}{\partial t_{k}}=\left(\left(B C^{-1}\right)^{k}\right)_{\geq 0} B-B\left(\left(C^{-1} B\right)^{k}\right)_{\geq 0}, \\
& \frac{\partial C}{\partial t_{k}}=\left(\left(B C^{-1}\right)^{k}\right)_{\geq 0} C-C\left(\left(C^{-1} B\right)^{k}\right)_{\geq 0}, \\
& \frac{\partial B}{\partial \bar{t}_{k}}=\left(\left(C B^{-1}\right)^{k}\right)_{<0} B-B\left(\left(B^{-1} C\right)^{k}\right)_{<0}, \\
& \frac{\partial C}{\partial \bar{t}_{k}}=\left(\left(C B^{-1}\right)^{k}\right)_{<0} C-C\left(\left(B^{-1} C\right)^{k}\right)_{<0} .
\end{aligned}
$$


These equations have another expression of the following form:

$$
\begin{aligned}
& \frac{\partial B}{\partial t_{k}}=-\left(\left(B C^{-1}\right)^{k}\right)_{<0} B+B\left(\left(C^{-1} B\right)^{k}\right)_{<0}, \\
& \frac{\partial C}{\partial t_{k}}=-\left(\left(B C^{-1}\right)^{k}\right)_{<0} C+C\left(\left(C^{-1} B\right)^{k}\right)_{<0}, \\
& \frac{\partial B}{\partial \bar{t}_{k}}=-\left(\left(C B^{-1}\right)^{k}\right)_{\geq 0} B+B\left(\left(B^{-1} C\right)^{k}\right)_{\geq 0}, \\
& \frac{\partial C}{\partial \bar{t}_{k}}=-\left(\left(C B^{-1}\right)^{k}\right)_{\geq 0} C+C\left(\left(B^{-1} C\right)^{k}\right)_{\geq 0} .
\end{aligned}
$$

We can deduce from these two expressions of (6.7) that the right hand side are difference operators of the form $f_{k}, g_{k} e^{-\partial_{s}}, \bar{f}_{k}$ and $\bar{g}_{k} e^{-\partial_{s}}$. Thus (6.7) can be reduced to evolution equations of the form

$$
\frac{\partial b}{\partial t_{k}}=f_{k}, \quad \frac{\partial c}{\partial t_{k}}=g_{k}, \quad \frac{\partial b}{\partial \bar{t}_{k}}=\bar{f}_{k}, \quad \frac{\partial c}{\partial \bar{t}_{k}}=\bar{g}_{k} .
$$

This implies that the factorized form (6.5) of the Lax operators is preserved by the flows of the 2D Toda hierarchy. (6.8) can be further converted to the usual Hamiltonian form [22] of the Ablowitz-Ladik hierarchy by a change of variables [24].

\section{Another formulation of reduction to Ablowitz-Ladik hierarchy}

The Lax operators of (6.5) have another expression

$$
L=\tilde{C}^{-1} \tilde{B}, \quad \bar{L}^{-1}=\tilde{B}^{-1} \tilde{C},
$$

where $\tilde{B}$ and $\tilde{C}$ are difference operators of the form

$$
\tilde{B}=e^{\partial_{s}}-\tilde{b}, \quad \tilde{C}=1-\tilde{c} e^{-\partial_{s}}
$$

that satisfies the relation

$$
\tilde{C} B=\tilde{B} C \text {. }
$$

$\tilde{B}^{-1}$ and $\tilde{C}^{-1}$ are understood to be defined by series expansions like (6.6). The operator relation (6.10) turns into the functional relations

$$
b(s)+\tilde{c}(s)=\tilde{b}(s)+c(s+1), \quad \tilde{c}(s) b(s-1)=\tilde{b}(s) c(s) .
$$


$b, c$ and $\tilde{b}, \tilde{c}$ are thus mutually connected by an invertible transformation. The Lax equations (6.1) can be reduced to the equations

$$
\begin{aligned}
& \left.\frac{\partial \tilde{B}}{\partial t_{k}}=\left(\left(\tilde{B} \tilde{C}^{-1}\right)^{k}\right)_{\geq 0} \tilde{B}-\tilde{B}\left(\tilde{C}^{-1} \tilde{B}\right)^{k}\right)_{\geq 0}, \\
& \frac{\partial \tilde{C}}{\partial t_{k}}=\left(\left(\tilde{B} \tilde{C}^{-1}\right)^{k}\right)_{\geq 0} \tilde{C}-\tilde{C}\left(\left(\tilde{C}^{-1} \tilde{B}\right)^{k}\right)_{\geq 0}, \\
& \frac{\partial \tilde{B}}{\partial \bar{t}_{k}}=\left(\left(\tilde{C} \tilde{B}^{-1}\right)^{k}\right)_{<0} \tilde{B}-\tilde{B}\left(\left(\tilde{B}^{-1} \tilde{C}\right)^{k}\right)_{<0}, \\
& \frac{\partial \tilde{C}}{\partial \bar{t}_{k}}=\left(\left(\tilde{C} \tilde{B}^{-1}\right)^{k}\right)_{<0} \tilde{C}-\tilde{C}\left(\left(\tilde{B}^{-1} \tilde{C}\right)^{k}\right)_{<0}
\end{aligned}
$$

for $\tilde{B}$ and $\tilde{C}$. These equations can be further converted to evolution equations for $\tilde{b}$ and $\tilde{c}$.

The factorized form (6.9) is suited for a comparison with the relativistic Toda hierarchy [16]. If the Lax operators are factorized in this form, the eigenvalue problem

$$
L \Psi=z \Psi, \quad \bar{L}^{-1} \bar{\Psi}=z^{-1} \bar{\Psi}
$$

for the Baker-Akhiezer functions $\Psi$ and $\bar{\Psi}$ of the 2D Toda hierarchy $[10,11]$ can be converted to a "generalized eigenvalue problem" of the form

$$
\tilde{B} \Psi=z \tilde{C} \Psi, \quad \tilde{B} \bar{\Psi}=z \tilde{C} \bar{\Psi} .
$$

It is this generalized eigenvalue problem that is used in Bruschi and Ragnisco's scalar-valued Lax formalism [23] of the relativistic Toda lattice. As pointed out by Kharchev et al. [21], this generalized eigenvalue problem can be derived from the traditional $2 \times 2$ matrix-valued Lax formalism [15] of the Ablowitz-Ladik hierarchy as well.

\subsection{Factorization problem of infinite matrices}

Difference operators in the Lax formalism of the 2D Toda hierarchy are in one-to-one correspondence with $\mathbb{Z} \times \mathbb{Z}$ matrices by the rule

$$
\sum_{k=-\infty}^{\infty} a_{k}(s) e^{k \partial_{s}} \longleftrightarrow \sum_{k=-\infty}^{\infty} \operatorname{diag}\left[a_{k}(s)\right] \Lambda^{k},
$$

where $\operatorname{diag}[a(s)]$ stands for the diagonal matrix

$$
\operatorname{diag}[a(s)]=\sum_{i, j \in \mathbb{Z}} a(i) E_{i i} .
$$


Since this correspondence preserves the algebraic structure, one can reformulate the Lax formalism of the $2 \mathrm{D}$ Toda hierarchy in terms of $\mathbb{Z} \times \mathbb{Z}$ matrices. Let us use the same notations $L, \bar{L}, B_{n}, \bar{B}_{n}$ etc. for the corresponding $\mathbb{Z} \times \mathbb{Z}$ matrices.

In this matrix formulation, one can formulate a matrix factorization problem that can capture all solutions of the 2D Toda hierarchy [26, 27, 28]. Given a constant invertible $\mathbb{Z} \times \mathbb{Z}$ matrix $U$, the problem is to find two $\mathbb{Z} \times \mathbb{Z}$ matrices $W=W(\boldsymbol{t}, \overline{\boldsymbol{t}})$ and $\bar{W}=\bar{W}(\boldsymbol{t}, \overline{\boldsymbol{t}})$ with the following properties:

(i) $W$ is a lower triangular matrix, and all diagonal elements are equal to 1 .

(ii) $\bar{W}$ is an upper triangular matrix, and all diagonal elements are nonzero.

(iii) $W$ and $\bar{W}$ satisfy the factorization relation

$$
\exp \left(\sum_{k=1}^{\infty} t_{k} \Lambda^{k}\right) U \exp \left(-\sum_{k=1}^{\infty} \bar{t}_{k} \Lambda^{-k}\right)=W^{-1} \bar{W} .
$$

If such matrices $W, \bar{W}$ exist, they satisfy the so called "Sato equations"

$$
\begin{aligned}
\frac{\partial W}{\partial t_{k}}=B_{k} W-W \Lambda^{k}, & \frac{\partial \bar{W}}{\partial t_{k}}=B_{k} \bar{W} \\
\frac{\partial W}{\partial \bar{t}_{k}}=\bar{B}_{k} W, & \frac{\partial \bar{W}}{\partial \bar{t}_{k}}=\bar{B}_{k} \bar{W}-\bar{W} \Lambda^{-k},
\end{aligned}
$$

where $B_{k}$ and $\bar{B}_{k}$ are constructed from $W$ and $\bar{W}$ as

$$
B_{k}=\left(W \Lambda^{k} W^{-1}\right)_{\geq 0}, \quad \bar{B}_{k}=\left(\bar{W} \Lambda^{-k} \bar{W}^{-1}\right)_{<0} .
$$

()$_{\geq 0}$ and ()$_{<0}$ stand for the upper triangular part and the strictly lower triangular part. The Sato equations (6.16) imply that the matrices $L$ and $\bar{L}$ defined by

$$
L=W \Lambda W^{-1}, \quad \bar{L}=\bar{W} \Lambda^{-1} \bar{W}^{-1}
$$

satisfy the Lax equations (6.1).

The factorization problem (6.15) is directly connected with the fermionic expression [13]

$$
\tau(s, \boldsymbol{t}, \overline{\boldsymbol{t}})=\left\langle s\left|\exp \left(\sum_{k=1}^{\infty} t_{k} J_{k}\right) g \exp \left(-\sum_{k=1}^{\infty} \bar{t}_{k} J_{-k}\right)\right| s\right\rangle
$$


of tau functions. Namely, the $\mathbb{Z} \times \mathbb{Z}$ matrix $U=\left(u_{i j}\right)$ corresponds to the operator $g$ by the Bogoliubov transformation

$$
g \psi_{j} g^{-1}=\sum_{i \in \mathbb{Z}} \psi_{i} u_{i j}
$$

of fermion creation-annihilation operators [12]. Its infinitesimal form is exactly the correspondence (5.1) between $\mathbb{Z} \times \mathbb{Z}$ matrices and fermion bilinears.

The operators (4.3) and (4.8) defining the tau functions $\tau(s, \boldsymbol{t})$ and $\tau^{\prime}(s, \boldsymbol{t}, \overline{\boldsymbol{t}})$ correspond to the matrices

$$
U=q^{\Delta^{2} / 2} \Gamma_{-}\left(q^{-\rho}\right) \Gamma_{+}\left(q^{-\rho}\right) Q^{\Delta} \Gamma_{-}\left(q^{-\rho}\right) \Gamma_{+}\left(q^{-\rho}\right) q^{\Delta^{2} / 2}
$$

and

$$
U^{\prime}=q^{\Delta^{2} / 2} \Gamma_{-}\left(q^{-\rho}\right) \Gamma_{+}\left(q^{-\rho}\right) Q^{\Delta} \Gamma_{-}^{\prime}\left(q^{-\rho}\right) \Gamma_{+}^{\prime}\left(q^{-\rho}\right) q^{-\Delta^{2} / 2},
$$

where $\Gamma_{ \pm}(\boldsymbol{x})$ and $\Gamma_{ \pm}^{\prime}(\boldsymbol{x})$ are the matrix representation (5.8) of the multivariable vertex operators. We now consider the factorization problem (6.15) in these particular cases.

\subsection{Initial values of Lax operators}

When $\boldsymbol{t}$ and $\overline{\boldsymbol{t}}$ are specialized to $\boldsymbol{t}=\overline{\boldsymbol{t}}=\mathbf{0}$, we can find an explicit form of the solution of the factorization problem (6.15) for the matrix (6.20):

Lemma 1. The factorization problem

$$
U^{\prime}=W^{-1} \bar{W}
$$

for the matrix (6.20) can be solved as

$$
\begin{gathered}
W=q^{\Delta^{2} / 2} \Gamma_{-}^{\prime}\left(Q q^{-\rho}\right)^{-1} \Gamma_{-}\left(q^{-\rho}\right)^{-1} q^{-\Delta^{2} / 2}, \\
\bar{W}=q^{\Delta^{2} / 2} Q^{\Delta} \Gamma_{+}\left(Q q^{-\rho}\right) \Gamma_{+}^{\prime}\left(q^{-\rho}\right) q^{-\Delta^{2} / 2} .
\end{gathered}
$$

Proof. We can use the algebraic relations

$$
Q^{\Delta} \Lambda^{n} Q^{-\Delta}=Q^{-n} \Lambda^{n}, \quad Q^{-\Delta} \Lambda^{n} Q^{\Delta}=Q^{n} \Lambda^{n}
$$

to rewrite the triple product in the middle of (6.20) as

$$
\begin{aligned}
\Gamma_{+}\left(q^{-\rho}\right) Q^{\Delta} \Gamma_{-}^{\prime}\left(q^{-\rho}\right) & =Q^{\Delta} \Gamma_{+}\left(Q q^{-\rho}\right) \Gamma_{-}^{\prime}\left(q^{-\rho}\right) \\
& =Q^{\Delta} \Gamma_{-}^{\prime}\left(q^{-\rho}\right) \Gamma_{+}\left(Q q^{-\rho}\right) \\
& =\Gamma_{-}^{\prime}\left(Q q^{-\rho}\right) Q^{\Delta} \Gamma_{+}\left(Q q^{-\rho}\right) .
\end{aligned}
$$


Thus $U^{\prime}$ becomes a product of triangular matrices:

$$
U^{\prime}=q^{\Delta^{2} / 2} \Gamma_{-}\left(q^{-\rho}\right) \Gamma_{-}^{\prime}\left(Q q^{-\rho}\right) \cdot Q^{\Delta} \Gamma_{+}\left(Q q^{-\rho}\right) \Gamma_{+}^{\prime}\left(q^{-\rho}\right) q^{-\Delta^{2} / 2} .
$$

We have only to modify the first (lower triangular) part with the diagonal matrix $q^{-\Delta^{2} / 2}$ so that all diagonal elements become 1 .

The initial values of the associated Lax operators (6.17) at $\boldsymbol{t}=\overline{\boldsymbol{t}}=\mathbf{0}$ are obtained from (6.22) as

$$
L=W \Lambda W^{-1}, \quad \bar{L}^{-1}=\bar{W} \Lambda^{-1} \bar{W}^{-1} .
$$

These matrices turn out to be a quotient of two matrices:

\section{Lemma 2.}

$$
L=\left(\Lambda-q^{\Delta}\right)\left(1+Q q^{\Delta-1} \Lambda^{-1}\right)^{-1}=\left(1+Q q^{\Delta} \Lambda^{-1}\right)^{-1}\left(\Lambda-q^{\Delta}\right) .
$$

Proof. By construction, $L$ is a matrix of the form

$$
L=q^{\Delta^{2} / 2} \Gamma_{-}^{\prime}\left(Q q^{-\rho}\right)^{-1} \Gamma_{-}\left(q^{-\rho}\right)^{-1} q^{-\Delta^{2} / 2} \Lambda q^{\Delta^{2} / 2} \Gamma_{-}\left(q^{-\rho}\right) \Gamma_{-}^{\prime}\left(Q q^{-\rho}\right) q^{-\Delta^{2} / 2} .
$$

Let us calculate this matrix step-by-step. Note that specialization of (5.11) to $k=0$ reads

$$
q^{\Delta^{2} / 2} \Lambda^{m} q^{-\Delta^{2} / 2}=q^{m^{2} / 2} \Lambda^{m} q^{-m \Delta} .
$$

This implies that

$$
q^{-\Delta^{2} / 2} \Lambda q^{\Delta^{2} / 2}=\Lambda q^{\Delta-1 / 2} .
$$

The next stage is to calculate the product

$$
\begin{aligned}
& \Gamma_{-}^{\prime}\left(Q q^{-\rho}\right)^{-1} \Gamma_{-}\left(q^{-\rho}\right)^{-1} q^{-\Delta^{2} / 2} \Lambda q^{\Delta^{2} / 2} \Gamma_{-}\left(q^{-\rho}\right) \Gamma_{-}^{\prime}\left(Q q^{-\rho}\right) \\
& =\Lambda \Gamma_{-}^{\prime}\left(Q q^{-\rho}\right)^{-1} \Gamma_{-}\left(q^{-\rho}\right)^{-1} q^{\Delta-1 / 2} \Gamma_{-}\left(q^{-\rho}\right) \Gamma_{-}^{\prime}\left(Q q^{-\rho}\right)
\end{aligned}
$$

with the aid of (5.12). Firstly, the triple product of $\Gamma_{-}\left(q^{-\rho}\right)^{ \pm 1}$ and $q^{\Delta}$ can be calculated as follows:

$$
\begin{aligned}
\Gamma_{-}\left(q^{-\rho}\right)^{-1} q^{\Delta} \Gamma_{-}\left(q^{-\rho}\right) & =\Gamma_{-}\left(q^{-\rho}\right)^{-1} \cdot q^{\Delta} \Gamma_{-}\left(q^{-\rho}\right) q^{-\Delta} \cdot q^{\Delta} \\
& =\prod_{i=1}^{\infty}\left(1-q^{i-1 / 2} \Lambda^{-1}\right) \cdot \prod_{i=1}^{\infty}\left(1-q^{i+1 / 2} \Lambda^{-1}\right)^{-1} \cdot q^{\Delta} \\
& =\left(1-q^{1 / 2} \Lambda^{-1}\right) q^{\Delta} .
\end{aligned}
$$


Since $1-q^{1 / 2} \Lambda^{-1}$ commutes with $\Gamma_{-}^{\prime}\left(Q q^{-\rho}\right)^{ \pm 1}$, the next task is to calculate a triple product of $\Gamma_{-}^{\prime}\left(Q q^{-\rho}\right)^{ \pm 1}$ and $q^{\Delta}$. This can be achieved as follows:

$$
\begin{aligned}
\Gamma_{-}^{\prime}\left(Q q^{-\rho}\right)^{-1} q^{\Delta} \Gamma_{-}^{\prime}\left(Q q^{-\rho}\right) & =\Gamma_{-}^{\prime}\left(Q q^{-\rho}\right)^{-1} \cdot q^{\Delta} \Gamma_{-}^{\prime}\left(Q q^{-\rho}\right) q^{-\Delta} \cdot q^{\Delta} \\
& =\prod_{i=1}^{\infty}\left(1+Q q^{i-1 / 2} \Lambda^{-1}\right)^{-1} \cdot \prod_{i=1}^{\infty}\left(1+Q q^{i+1 / 2} \Lambda^{-1}\right) \cdot q^{\Delta} \\
& =\left(1+Q q^{1 / 2} \Lambda^{-1}\right)^{-1} q^{\Delta} .
\end{aligned}
$$

These calculations yield the equality

$$
\begin{array}{r}
\Gamma_{-}^{\prime}\left(Q q^{-\rho}\right)^{-1} \Gamma_{-}\left(q^{-\rho}\right)^{-1} q^{-\Delta^{2} / 2} \Lambda q^{\Delta^{2} / 2} \Gamma_{-}\left(q^{-\rho}\right) \Gamma_{-}^{\prime}\left(Q q^{-\rho}\right) \\
=\Lambda\left(1-q^{1 / 2} \Lambda^{-1}\right)\left(1+Q q^{1 / 2} \Lambda^{-1}\right)^{-1} q^{\Delta-1 / 2},
\end{array}
$$

hence an expression of $L$ of the form

$$
L=q^{\Delta^{2} / 2}\left(\Lambda-q^{1 / 2}\right)\left(1+Q q^{1 / 2} \Lambda^{-1}\right) q^{\Delta-1 / 2} q^{-\Delta^{2} / 2} .
$$

We now use the algebraic relations

$$
\begin{aligned}
& q^{\Delta^{2} / 2} \Lambda q^{-\Delta^{2} / 2}=q^{-\Delta-1 / 2} \Lambda, \quad q^{\Delta^{2} / 2} \Lambda^{-1} q^{-\Delta^{2} / 2}=q^{\Delta-1 / 2} \Lambda^{-1} \\
& \Lambda q^{\Delta}=q^{\Delta+1} \Lambda, \quad \Lambda^{-1} q^{\Delta}=q^{\Delta-1} \Lambda^{-1}
\end{aligned}
$$

that follow from (6.24) and (5.12) to rewrite $L$ further as follows:

$$
\begin{aligned}
L & =\left(q^{-\Delta-1 / 2} \Lambda-q^{1 / 2}\right)\left(1+Q q^{\Delta} \Lambda^{-1}\right) q^{\Delta-1 / 2} \\
& =q^{\Delta-1 / 2}\left(q^{-\Delta+1 / 2} \Lambda-q^{1 / 2}\right)\left(1+Q q^{\Delta-1} \Lambda^{-1}\right)^{-1} \\
& =\left(\Lambda-q^{\Delta}\right)\left(1+Q q^{\Delta-1} \Lambda^{-1}\right)^{-1} .
\end{aligned}
$$

This gives the first part of (6.23). In the same way, starting from

$$
L=q^{\Delta^{2} / 2}\left(1+Q q^{1 / 2} \Lambda^{-1}\right)^{-1}\left(\Lambda-q^{1 / 2}\right) q^{\Delta-1 / 2} q^{-\Delta^{2} / 2}
$$

and repeating similar calculations, one can derive the second part of (6.23).

\section{Lemma 3.}

$$
\bar{L}^{-1}=\left(1+Q q^{\Delta-1} \Lambda^{-1}\right)\left(q^{\Delta}-\Lambda\right)^{-1}=\left(q^{\Delta}-\Lambda\right)^{-1}\left(1+Q q^{\Delta} \Lambda^{-1}\right) .
$$

Proof. The proof is parallel to the case of the previous lemma. By construction, $\bar{L}^{-1}$ is a matrix of the form

$$
\begin{aligned}
\bar{L}^{-1}=q^{\Delta^{2} / 2} & Q^{\Delta} \Gamma_{+}\left(Q q^{-\rho}\right) \Gamma_{+}^{\prime}\left(q^{-\rho}\right) q^{-\Delta^{2} / 2} \Lambda^{-1} q^{\Delta^{2} / 2} \\
& \times \Gamma_{+}^{\prime}\left(q^{-\rho}\right)^{-1} \Gamma_{+}\left(Q q^{-\rho}\right)^{-1} Q^{-\Delta} q^{-\Delta^{2} / 2} .
\end{aligned}
$$


With the aid of (6.24) and (5.12), one can derive the equality

$$
\begin{gathered}
\Gamma_{+}\left(Q q^{-\rho}\right) \Gamma_{+}^{\prime}\left(q^{-\rho}\right) q^{-\Delta^{2} / 2} \Lambda^{-1} q^{\Delta^{2} / 2} \Gamma_{+}^{\prime}\left(q^{-\rho}\right)^{-1} \Gamma_{+}\left(Q q^{-\rho}\right)^{-1} \\
=\Lambda^{-1}\left(1+q^{1 / 2} \Lambda\right)\left(1-Q q^{1 / 2} \Lambda\right)^{-1} q^{-\Delta-1 / 2} .
\end{gathered}
$$

This implies that

$$
\bar{L}^{-1}=q^{\Delta^{2} / 2} Q^{\Delta}\left(1+q^{-1 / 2} \Lambda^{-1}\right)\left(1-Q q^{1 / 2} \Lambda\right)^{-1} q^{-\Delta} Q^{-\Delta} q^{-\Delta^{2} / 2} .
$$

It is easy to derive the final form (6.25) of $\bar{L}^{-1}$ from this expression.

\subsection{Identification of solution}

Translated to the language of difference operators, the foregoing results (6.23) and (6.25) show that the initial values of the Lax operators at $\boldsymbol{t}=\overline{\boldsymbol{t}}=\mathbf{0}$ are factorized as

$$
L=B C^{-1}=\tilde{C}^{-1} \tilde{B}, \quad \bar{L}^{-1}=-C B^{-1}=-\tilde{B}^{-1} \tilde{C}
$$

with factors of the special form

$$
B=\tilde{B}=e^{\partial_{s}}-q^{s}, \quad C=1+Q q^{s-1} e^{-\partial_{s}}, \quad \tilde{C}=1+Q q^{s} e^{-\partial_{s}} .
$$

Note that this expression is slightly different from (6.5) and (6.9). Namely, $\bar{L}^{-1}$ has an extra negative sign. This does not affect the essential part of the reduction procedure in the case of (6.5). The outcome of this procedure is the "twisted" version

$$
\frac{\partial b}{\partial t_{k}}=f_{k}, \quad \frac{\partial c}{\partial t_{k}}=g_{k}, \quad(-1)^{k} \frac{\partial b}{\partial \bar{t}_{k}}=\bar{f}_{k}, \quad(-1)^{k} \frac{\partial c}{\partial \bar{t}_{k}}=\bar{g}_{k}
$$

of the evolution equations (6.8). The reduced system is the same AblowitzLadik hierarchy except that the second set of time variables $\overline{\boldsymbol{t}}$ are replaced by $\iota(\overline{\boldsymbol{t}})=\left(-t_{1}, t_{2},-t_{3}, \ldots\right)$.

Thus we can conclude that the factorized Lax operators (6.26) with the initial data (6.27) at $\boldsymbol{t}=\overline{\boldsymbol{t}}=\mathbf{0}$ persist to be factorized after time evolutions. In summary, we have proven the following proposition:

Theorem 4. The factorization problem (6.15) for the matrix (6.20) yields a solution of the 2D Toda hierarchy with the tau function (4.10). The associated Lax operators $L$ and $\bar{L}$ are factorized in the form of (6.26). In particular, this is a solution of the Ablowitz-Ladik hierarchy with the second set of time variables $\overline{\boldsymbol{t}}$ being replaced by $\iota(\overline{\boldsymbol{t}})$. 
Remark 3. Unlike the reduction condition (6.5) of Brini et al. [17, 24], our reduction condition (6.26) has extra negative signs. This apparent discrepancy seems to be related to the fact that the usual expression $[6,8]$ of the string amplitude $Z_{X_{1}}$ of the resolved conifold contains the parameter $Q$ with with a negative sign:

$$
Z_{X_{1}}=\sum_{\lambda \in \mathcal{P}} s_{\lambda}\left(q^{-\rho}\right) s_{{ } \lambda}\left(q^{-\rho}\right)(-Q)^{|\lambda|} .
$$

Remark 4. The factorization problem (6.15) for the matrix (6.19), too, can be treated in the same way. The solution at $\boldsymbol{t}=\overline{\boldsymbol{t}}=\mathbf{0}$ reads

$$
\begin{gathered}
W=q^{\Delta^{2} / 2} \Gamma_{-}\left(Q q^{-\rho}\right)^{-1} \Gamma_{-}\left(q^{-\rho}\right)^{-1} q^{-\Delta^{2} / 2}, \\
\bar{W}=q^{\Delta^{2} / 2} Q^{\Delta} \Gamma_{+}\left(Q q^{-\rho}\right) \Gamma_{+}\left(q^{-\rho}\right) q^{\Delta^{2} / 2} .
\end{gathered}
$$

The initial values of the associated Lax operators at $\boldsymbol{t}=\overline{\boldsymbol{t}}=\mathbf{0}$ have the factorized form

$$
L=\bar{L}^{-1}=e^{\partial_{s}}\left(1-q^{s-1} e^{-\partial_{s}}\right)\left(1-Q q^{s-1} e^{-\partial_{s}}\right) .
$$

Such a factorized form is preserved by the flows of the 2D Toda hierarchy.

\section{Acknowledgements}

This work is partly supported by JSPS Grants-in-Aid for Scientific Research No. 21540218 and No. 22540186 from the Japan Society for the Promotion of Science.

\section{References}

[1] T. Nakatsu and K. Takasaki, Melting crystal, quantum torus and Toda hierarchy, Commun. Math. Phys. 285 (2009), 445-468, arXiv:0710.5339 [hep-th].

[2] T. Nakatsu and K. Takasaki, Integrable structure of melting crystal model with external potentials, Adv. Stud. Pure Math. vol. 59 (Mathematical Society of Japan, Tokyo, 2010), pp. 201-223, arXiv:0807.4970 [math-ph].

[3] A. Okounkov, N. Reshetikhin and C. Vafa, Quantum Calabi-Yau and classical crystals, P. Etingof, V. Retakh and I.M. Singer (eds.), The unity of mathematics, Progr. Math. vol. 244 (Birkhäuser, 2006), pp. 597-618, arXiv:hep-th/0309208 
[4] T. Maeda, T. Nakatsu, K. Takasaki and T. Tamakoshi, Five-dimensional supersymmetric Yang-Mills theories and random plane partitions, JHEP 0503 (2005), 056, arXiv:hep-th/0412327.

[5] K. Takasaki, Integrable structure of modified melting crystal model, arXiv:1208.4497 [math-ph].

[6] M. Aganagic, A. Klemm, M. Mariño and C. Vafa, The topological vertex, Commun. Math. Phys. 254 (2005), 425-478, arXiv:hep-th/0305132.

[7] J. Bryan and R. Pandharipande, The local Gromov-Witten theory of curves, J. Amer. Math. Soc. 21 (2008), 101-136, arXiv:math/0411037.

[8] N. Caporaso, L. Griguolo, M. Mariño, S. Pasquetti and D. Seminara, Phase transitions, double-scaling limit, and topological strings, Phys. Rev. D75 (2007), 046004, arXiv:hep-th/0606120.

[9] A. Okounkov and N. Reshetikhin, Correlation function of Schur process with application to local geometry of a random 3dimensional young diagram, J. Amer. Math. Soc. 16, (2003), 581-603, arXiv:math.CO/0107056

[10] K. Ueno and K. Takasaki, Toda lattice hierarchy, Adv. Stud. Pure Math. vol. 4 (Kinokuniya, Tokyo, 1984), pp. 1-95.

[11] K. Takasaki and T. Takebe, Integrable hierarchies and dispersionless limit, Rev. Math. Phys. 7 (1995), 743-808, arXiv:hep-th/9405096.

[12] T. Miwa, M. Jimbo and E. Date, Solitons: Differential equations, symmetries, and infinite-dimensional algebras, Cambridge University Press, 2000 .

[13] T. Takebe, Representation theoretical meanings of the initial value problem for the Toda lattice hierarchy I, Lett. Math. Phys. 21 (1991), 77-84.

[14] J. Bryan and B. Young, Generating functions for coloured 3D Young diagrams and the Donaldson-Thomas invariants of orbifolds, Duke Math. J. 152 (2010), 115-153, arXiv:0802.3948 [math.CO].

[15] M. J. Ablowitz and J. F. Ladik, Nonlinear differential-difference equations, J. Math. Phys. 16 (1975), 598-603.

[16] S. N. M. Ruijsenaars, Relativistic Toda systems, Commun. Math. Phys. 133 (1990), 217-247. 
[17] A. Brini, The local Gromov-Witten theory of $\mathbb{C P}^{1}$ and integrable hierarchies, Commun. Math. Phys. 313 (2012), 571-605, arXiv:1002.0582 [math-ph].

[18] V. E. Vekslerchik, Functional representation of the Ablowitz-Ladik hierarchy, J. Phys. A: Math. Gen. 31 (1998), 1087-1099, arXiv:solvint/9707008.

[19] M. Adler and P. van Moerbeke, Integrals over classical groups, random permutations, Toda and Toeplitz lattices, Comm. Pure Appl. Math. 54 (2001), 153-205, arXiv:math/9912143.

[20] M. Cafasso, Matrix biorthogonal polynomials on the unit circle and nonabelian Ablowitz-Ladik hierarchy, J. Phys. A: Math. Theor. 42 (2009), 365211, arXiv:0804.3572 [math.CA].

[21] S. Kharchev, A. Mironov and A. Zhedanov, Faces of relativistic Toda chain, Int. J. Mod. Phys. A12 (1997), 2675-2724, arXiv:hepth/9606144.

[22] Yu. B. Suris, A note on the integrable discretization of the nonlinear Schrödinger equation, Inverse Problems 13 (1997), 1121-1136, arXiv:solv-int/9701010.

[23] M. Bruschi and O. Ragnisco, Lax representation and complete integrability for the periodic relativistic Toda lattice, Phys. Lett. A134 (1989), 365-370.

[24] A. Brini, G. Carlet and P. Rossi, Integrable hierarchies and the mirror model of local $\mathbb{C P}^{1}$, Physica D241 (2012), 2156-2167. arXiv:1105.4508 [math.AG].

[25] I. M. Krichever, General rational reductions of the KadomtsevPetviashvili hierarchy and their symmetries, Funct. Anal. Appl. 29 (2) (1995), 75-80.

[26] K. Takasaki, Initial value problem for the Toda lattice hierarchy, Adv. Stud. Pure Math. vol. 4 (Kinokuniya, Tokyo, 1984), pp. 136-163.

[27] T. Nakatsu, K. Takasaki and S. Tsujimaru, Quantum and classical aspects of deformed $c=1$ strings, Nucl. Phys. B443 (1995), 1550-197, arXiv:hep-th/9501038.

[28] K. Takasaki, Toda lattice hierarchy and generalized string equations, Commun. Math. Phys. 181 (1996), 131-156, arXiv:hep-th/9506089. 
[29] L. Faddeev and A. Yu. Volkov, Abelian current algebra and the Virasoro algebra on the lattice, Phys. Lett. B315 (1993), 311-318, arXiv:hepth/9307048.

[30] L. D. Faddeev and R. M. Kashaev, Quantum dilogarithm, Mod. Phys. Lett. A9 (1994), 427-434. arXiv:hep-th/9310070.

[31] I. G. Macdonald, Symmetric functions and Hall polynomials, Oxford University Press, 1995. 